\title{
Isospin-invariant Skyrme energy-density-functional approach with axial symmetry
}

\author{
J. A. Sheikh, ${ }^{1,2,3}$ N. Hinohara, ${ }^{1,2,4}$ J. Dobaczewski, ${ }^{1,5,6}$ T. Nakatsukasa, ${ }^{7,8}$ W. Nazarewicz, ${ }^{1,5,9}$ and K. Sato ${ }^{7}$ \\ ${ }^{1}$ Department of Physics and Astronomy, University of Tennessee, Knoxville, Tennessee 37996-1200, USA \\ ${ }^{2}$ Joint Institute for Heavy-Ion Research, Oak Ridge, Tennessee 37831-6374, USA \\ ${ }^{3}$ Department of Physics, University of Kashmir, Srinagar, 190 006, India \\ ${ }^{4}$ Department of Physics and Astronomy, University of North Carolina, Chapel Hill, North Carolina 27599-3255, USA \\ ${ }^{5}$ Institute of Theoretical Physics, Faculty of Physics, University of Warsaw, Hoza 69, 00-681 Warsaw, Poland \\ ${ }^{6}$ Department of Physics, P.O. Box 35 (YFL) University of Jyväskylä, FI-40014 Jyväskylä, Finland \\ ${ }^{7}$ RIKEN Nishina Center, Wako 351-0198, Japan \\ ${ }^{8}$ Center for Computational Sciences, University of Tsukuba, Tsukuba 305-8571, Japan \\ ${ }^{9}$ Physics Division, Oak Ridge National Laboratory, P.O. Box 2008, Oak Ridge, Tennessee 37831-6373, USA
}

(Received 10 March 2014; revised manuscript received 21 April 2014; published 19 May 2014)

\begin{abstract}
Background: Density functional theory (DFT) is the microscopic tool of choice to describe properties of nuclei over the entire nuclear landscape, with a focus on medium-mass and heavy complex systems. Modern energy density functionals (EDFs) often offer a level of accuracy typical of phenomenological approaches based on parameters locally fitted to the data. It is clear, however, that in order to achieve high quality of predictions to guide spectroscopic studies, current functionals must be improved, especially in the isospin channel. In this respect, experimental studies of short-lived nuclei far from stability offer a unique test of isospin aspects of the many-body theory.

Purpose: We develop the isospin-invariant Skyrme-EDF method by considering local densities in all possible isospin channels and proton-neutron $(p-n)$ mixing terms as mandated by the isospin symmetry. The EDF employed has the most general form that depends quadratically on the isoscalar and isovector densities. We test and benchmark the resulting $p-n$ EDF approach, and study the general properties of the new scheme by means of the cranking in the isospin space.

Methods: We extend the existing axial DFT solver HFBTHO to the case of isospin-invariant EDF approach with all possible $p-n$ mixing terms. Explicit expressions have been derived for all the densities and potentials that appear in the isospin representation. In practical tests, we consider the Skyrme EDF SkM* and, as a first application, concentrate on Hartree-Fock aspects of the problem, i.e., pairing has been disregarded.

Results: Calculations have been performed for the $(A=78, T \simeq 11),(A=40, T \simeq 8)$, and $(A=48, T \simeq 4)$ isobaric analog chains. Isospin structure of self-consistent $p-n$ mixed solutions has been investigated with and without the Coulomb interaction, which is the sole source of isospin symmetry breaking in our approach. The extended axial HFBTHO solver has been benchmarked against the symmetry-unrestricted HFODD code for deformed and spherical states.

Conclusions: We developed and tested a general isospin-invariant Skyrme-EDF framework. The new approach permits spin-isospin densities that may give rise to hitherto unexplored modes in the excitation spectrum. The new formalism has been tested in the Hartree-Fock limit. A systematic comparison between HFODD and HFBTHO results show a maximum deviation of about $10 \mathrm{keV}$ on the total binding energy for deformed nuclei when the Coulomb term is included. Without this term, the results of both solvers agree down to a $\sim 10 \mathrm{eV}$ level.
\end{abstract}

DOI: 10.1103/PhysRevC.89.054317

\section{INTRODUCTION}

A major challenge for low-energy nuclear theory is to develop a universal nuclear EDF that can be used to explain and predict static and dynamic properties of atomic nuclei throughout the entire nuclear landscape within the framework of nuclear DFT. In a worldwide effort to develop a generalpurpose nuclear EDF [1-3], various strategies are applied, and, to realize this vision, the properties of rare isotopes are an essential guide.

In the quest of developing a universal nuclear EDF, the existing functionals ought to be enriched by incorporating the neglected couplings, especially in the spin and isospin channels. Indeed, the recent work [4] suggests that the Skyrme EDF has reached its limits and significant changes to the form
PACS number(s): 21.10.Hw, 21.60.Jz, 21.10.Sf

of the functional are needed. As far as the isospin sector is concerned, most of the EDFs include isoscalar particle densities and a single $t_{z}$ component in the isovector channel. The $t_{x}$ and $t_{y}$, or $p-n$ mixed, components of isovector densities are completely neglected. In the heavier nuclei where neutrons and protons occupy different shell-model spaces, the neglect of the $p-n$ mixed densities could be justified. However, in the lighter and medium-mass nuclei, neutrons and protons move in the same shells and the exclusion of these isovector densities cannot be justified. There are several observations that indicate deficiencies inherent in the existing EDF and other approaches to describe nuclei in the vicinity of the $N=Z$ line [5,6]. For instance, it is quite well established that binding energies of the nuclei close to the $N=Z$ line are underestimated by 
theoretical models [7,8], and $p-n$ correlations are expected to be the missing piece of physics in this puzzle.

In some earlier studies, $p-n$ mixing has been investigated in the particle-particle channel [9-14] (see Refs. [5,6] and Sec. IV of Ref. [15] for a more complete list of references). In the particle-hole sector, however, the $p-n$ mixing and resulting symmetry breaking effects have been largely neglected (a notable exception is the recent study of ${ }^{21} \mathrm{Ne}$ [16] that considered a $p$ - $n$ mixing on the HF level). As discussed in [15], such an approximation does not seem to be justified as the self-consistent polarization between particle-hole (p-h) and particle-particle (p-p) HFB channels is known to be strong. In Refs. [15,17] a generalized EDF approach has been proposed that allows for the arbitrary mixing of protons and neutrons, and an isospin-invariant EDF has been constructed. It has been shown that the generalized EDF gives rise to novel spin-isospin combinations of nucleonic densities that are absent in the standard Skyrme approaches. We expect that those extensions may lead to new, hitherto unexplored, nuclear modes.

The main objective of this study is to develop, test, and benchmark the isospin-invariant Skyrme-EDF (pnEDF) approach formulated in the cylindrical coordinate system, whose building blocks are all possible $p$ - $n$ mixed local densities. Since the majority of nuclei are axial in their ground states, such an approach will allow us to extend the global surveys of nuclear properties [18-21] made with the axial DFT solver HFBTHO [22,23] to observables and decays related to isospin. The code HFBTHO has been optimized for performance on flagship computing platforms, for it serves as a backbone of the EDF optimization package [4,24]. In a parallel study [25], $p-n$ mixed densities have also been implemented in the general-purpose solver HFODD [26] written in a three-dimensional Cartesian basis. We take advantage of this development to benchmark both pnEDF schemes.

The paper is organized as follows. Basic expressions pertaining to the isospin-invariant pnEDF approach are briefly summarized in Sec. II. Section III discusses the HF application of the formalism to isobaric analog states (IASs) using the two-dimensional isocranking formalism. The axial HFBTHO pnEDF framework is benchmarked against the symmetryunconstrained pnEDF HFODD approach in Sec. IV. Finally, Sec. V contains the summary of our work and prospects for further developments.

\section{BASICS OF pnEDF APPROACH}

The pnEDF Kohn-Sham state $|\Psi\rangle$ is a single Slater determinant built of the set of $A$ fully occupied single-particle (s.p.) states, that is,

$$
|\Psi\rangle=\prod_{k=1}^{A} c_{k}^{+}|0\rangle,
$$

where $c_{k}^{+}$denotes the s.p. state creation operator. This operator can be expressed in terms of the s.p. wave function $V_{k}(\boldsymbol{r} s t)$,

$$
c_{k}^{+}=\int d^{3} \boldsymbol{r} \sum_{s t} V_{k}^{*}(\boldsymbol{r} s t) a_{\boldsymbol{r} s t}^{+} \quad(k \leqslant A),
$$

where $a_{r s t}^{+}$creates the nucleon at point $\boldsymbol{r}$, spin $s= \pm \frac{1}{2}$, and isospin $t=+\frac{1}{2}$ (neutron) or $-\frac{1}{2}$ (proton). In the $p$ - $n$ mixing framework, the HF s.p. state $c_{k}^{+}$contains the neutron and proton components. In the present study, we only consider unpaired systems and particle-hole (p-h) densities, whereupon $V_{k}(\boldsymbol{r} s t)$ are simply the self-consistent HF wave functions. However, expressions given below are also valid within the HFB approach, where $V_{k}(\boldsymbol{r} s t)$ correspond to lower components of the quasiparticle wave functions $[27,28]$.

To fix the notation, we now recall basic expressions introduced and derived in Ref. [17]. The one-body density matrix $\hat{\rho}$ is defined as

$$
\begin{aligned}
\hat{\rho}\left(\boldsymbol{r} s t, \boldsymbol{r}^{\prime} s^{\prime} t^{\prime}\right) & =\left\langle\Psi\left|a_{\boldsymbol{r}^{\prime} s^{\prime} t^{\prime}}^{+} a_{\boldsymbol{r} s t}\right| \Psi\right\rangle \\
& =\sum_{k=1}^{A} V_{k}\left(\boldsymbol{r}^{\prime} s^{\prime} t^{\prime}\right) V_{k}^{*}(\boldsymbol{r} s t),
\end{aligned}
$$

and the pnEDF can be written as

$$
\bar{H}[\hat{\rho}]=\int d^{3} \boldsymbol{r} \mathcal{H}(\boldsymbol{r})=\int d^{3} \boldsymbol{r} \mathcal{H}_{\mathrm{Sk}}(\boldsymbol{r})+E_{\mathrm{Cou}}[\hat{\rho}],
$$

where the Skyrme energy density is

$$
\mathcal{H}_{\mathrm{Sk}}(\boldsymbol{r})=\frac{\hbar^{2}}{2 m} \tau_{0}(\boldsymbol{r})+\chi_{0}(\boldsymbol{r})+\chi_{1}(\boldsymbol{r})
$$

with $\tau_{0}(\boldsymbol{r})$ being the isoscalar kinetic-energy density (it is assumed in the following that the neutron and proton masses are equal). The Coulomb energy functional $E_{\text {Cou }}$ is the only term that breaks the isospin symmetry. The Slater approximation is used for the Coulomb exchange functional. The p-h Skyrme interaction-energy densities $\chi_{0}(\boldsymbol{r})$ and $\chi_{1}(\boldsymbol{r})$ depend quadratically on the isoscalar and isovector densities, respectively. Based on general rules of constructing the energy density [17], one obtains

$$
\begin{aligned}
\chi_{0}(\boldsymbol{r})= & C_{0}^{\rho} \rho_{0}^{2}+C_{0}^{\Delta \rho} \rho_{0} \Delta \rho_{0}+C_{0}^{\tau} \rho_{0} \tau_{0}+C_{0}^{J 0} J_{0}^{2}+C_{0}^{J 1} \boldsymbol{J}_{0}^{2}+C_{0}^{J 2} \underline{J}_{0}^{2}+C_{0}^{\nabla J} \rho_{0} \nabla \cdot \boldsymbol{J}_{0} \\
& +C_{0}^{s} \boldsymbol{s}_{0}^{2}+C_{0}^{\Delta s} \boldsymbol{s}_{0} \cdot \Delta \boldsymbol{s}_{0}+C_{0}^{T} \boldsymbol{s}_{0} \cdot \boldsymbol{T}_{0}+C_{0}^{j} \boldsymbol{j}_{0}^{2}+C_{0}^{\nabla j} \boldsymbol{s}_{0} \cdot\left(\nabla \times \boldsymbol{j}_{0}\right)+C_{0}^{\nabla s}\left(\nabla \cdot \boldsymbol{s}_{0}\right)^{2}+C_{0}^{F} \boldsymbol{s}_{0} \cdot \boldsymbol{F}_{0}, \\
\chi_{1}(\boldsymbol{r})= & C_{1}^{\rho} \vec{\rho}^{2}+C_{1}^{\Delta \rho} \vec{\rho} \circ \Delta \vec{\rho}+C_{1}^{\tau} \vec{\rho} \circ \vec{\tau}+C_{1}^{J 0} \vec{J}^{2}+C_{1}^{J 1} \overrightarrow{\boldsymbol{J}}^{2}+C_{1}^{J 2} \underline{\boldsymbol{j}}^{2}+C_{1}^{\nabla J} \vec{\rho} \circ \nabla \cdot \overrightarrow{\boldsymbol{J}} \\
& +C_{1}^{s} \overrightarrow{\boldsymbol{s}}^{2}+C_{1}^{\Delta s} \overrightarrow{\boldsymbol{s}} \cdot \circ \Delta \overrightarrow{\boldsymbol{s}}+C_{1}^{T} \overrightarrow{\boldsymbol{s}} \cdot \circ \overrightarrow{\boldsymbol{T}}+C_{1}^{j} \overrightarrow{\boldsymbol{j}}^{2}+C_{1}^{\nabla j} \overrightarrow{\boldsymbol{s}} \cdot \circ(\nabla \times \overrightarrow{\boldsymbol{j}})+C_{1}^{\nabla s}(\nabla \cdot \overrightarrow{\boldsymbol{s}})^{2}+C_{1}^{F} \overrightarrow{\boldsymbol{s}} \cdot \circ \overrightarrow{\boldsymbol{F}},
\end{aligned}
$$


where $\times$ stands for the vector product of vectors in space, o stands for the scalar product of isovectors in isospace, and other definitions closely follow those introduced in Ref. [17].

Quasilocal densities $\rho_{k}, \tau_{k}, \boldsymbol{s}_{k}, \boldsymbol{T}_{k}, \boldsymbol{j}_{k}, \boldsymbol{F}_{k}, \boldsymbol{J}_{k}, \boldsymbol{J}_{k}$, and $\underline{J}_{k}$, are defined through the particle and spin nonlocal densities,

$$
\begin{aligned}
& \rho_{k}\left(\boldsymbol{r}, \boldsymbol{r}^{\prime}\right)=\sum_{s t t^{\prime}} \hat{\rho}\left(\boldsymbol{r} s t, \boldsymbol{r}^{\prime} s t^{\prime}\right) \hat{\tau}_{t^{\prime} t}^{k}, \\
& \boldsymbol{s}_{k}\left(\boldsymbol{r}, \boldsymbol{r}^{\prime}\right)=\sum_{s s^{\prime} t t^{\prime}} \hat{\rho}\left(\boldsymbol{r} s t, \boldsymbol{r}^{\prime} s^{\prime} t^{\prime}\right) \hat{\boldsymbol{\sigma}}_{s^{\prime} s} \hat{\tau}_{t^{\prime} t}^{k},
\end{aligned}
$$

where $k$ runs from 0 to $3, \hat{\sigma}$ and $\hat{\tau}^{k}(k=1,2,3)$ are the Pauli matrices for spin and isospin, respectively, and $\hat{\tau}_{t^{\prime} t}^{0}=\delta_{t^{\prime} t}$. The explicit definitions and expressions in the cylindrical basis for the local densities appearing in Eqs. (6) are given in the Appendix. By varying the pnEDF with respect to the density matrices, one obtains the p-h mean-field Hamiltonian:

$$
\begin{aligned}
\hat{h}\left(\boldsymbol{r}^{\prime} s^{\prime} t^{\prime}, \boldsymbol{r} s t\right)= & \frac{\delta \bar{H}[\hat{\rho}]}{\delta \hat{\rho}\left(\boldsymbol{r} s t, \boldsymbol{r}^{\prime} s^{\prime} t^{\prime}\right)} \\
= & -\frac{\hbar^{2}}{2 m} \delta\left(\boldsymbol{r}-\boldsymbol{r}^{\prime}\right) \nabla \cdot \nabla \delta_{s^{\prime} s} \delta_{t^{\prime} t} \\
& +\hat{\Gamma}\left(\boldsymbol{r}^{\prime} s^{\prime} t^{\prime}, \boldsymbol{r} s t\right)+\hat{\Gamma}_{\mathrm{r}}\left(\boldsymbol{r}^{\prime} s^{\prime} t^{\prime}, \boldsymbol{r} s t\right),
\end{aligned}
$$

where $\hat{\Gamma}$ is the HF potential and $\hat{\Gamma}_{\mathrm{r}}$ is the rearrangement potential.

For the pnEDF depending on quasilocal densities only, such as in Eq. (6), the HF Hamiltonian is a local differential operator,

$$
\hat{h}\left(\boldsymbol{r}^{\prime} s^{\prime} t^{\prime}, \boldsymbol{r} s t\right)=\delta\left(\boldsymbol{r}-\boldsymbol{r}^{\prime}\right) \hat{h}\left(\boldsymbol{r} ; s^{\prime} t^{\prime}, s t\right),
$$

which has a simple isospin structure:

$$
\hat{h}\left(\boldsymbol{r} ; s^{\prime} t^{\prime}, s t\right)=h_{0}\left(\boldsymbol{r} ; s^{\prime}, s\right) \delta_{t^{\prime} t}+\vec{h}\left(\boldsymbol{r} ; s^{\prime}, s\right) \circ \hat{\vec{\tau}}_{t^{\prime} t} .
$$

The isoscalar and isovector parts of the HF Skyrme Hamiltonian can be written in the compact form as

$$
\begin{aligned}
h_{k}\left(\boldsymbol{r} ; s^{\prime}, s\right)= & -\frac{\hbar^{2}}{2 m} \nabla^{2} \delta_{s^{\prime} s} \delta_{k 0}+U_{k} \delta_{s^{\prime} s}+\boldsymbol{\Sigma}_{k} \cdot \hat{\boldsymbol{\sigma}}_{s^{\prime} s} \\
& +\frac{1}{2 i}\left[\boldsymbol{I}_{k} \delta_{s^{\prime} s}+\left(\mathrm{B}_{k} \cdot \hat{\boldsymbol{\sigma}}_{s^{\prime} s}\right)\right] \cdot \nabla \\
& +\frac{1}{2 i} \nabla \cdot\left[\boldsymbol{I}_{k} \delta_{s^{\prime} s}+\left(\mathrm{B}_{k} \cdot \hat{\boldsymbol{\sigma}}_{s^{\prime} s}\right)\right] \\
& -\nabla \cdot\left[M_{k} \delta_{s^{\prime} s}+\boldsymbol{C}_{k} \cdot \hat{\boldsymbol{\sigma}}_{s^{\prime} s}\right] \nabla \\
& -\nabla \cdot \boldsymbol{D}_{k} \hat{\boldsymbol{\sigma}}_{s^{\prime} s} \cdot \nabla,
\end{aligned}
$$

where $k=0,1,2,3$, and

$$
(\mathrm{B} \cdot \hat{\boldsymbol{\sigma}})_{a}=\sum_{b} \mathrm{~B}_{a b} \hat{\boldsymbol{\sigma}}^{b}
$$

for $a=x, y, z$, denotes the $a$ th component of a space vector. By introducing the unit space tensor $\delta$ and the antisymmetric space tensor $(\boldsymbol{\epsilon} \cdot \boldsymbol{J})_{a b}=\sum_{c} \boldsymbol{\epsilon}_{a c b} \boldsymbol{J}_{c}$, the local real potentials can be written as

$$
\begin{aligned}
U_{k}(\boldsymbol{r})= & 2 C_{t}^{\rho} \rho_{k}+2 C_{t}^{\Delta \rho} \Delta \rho_{k}+C_{t}^{\tau} \tau_{k}+C_{t}^{\nabla J} \nabla \cdot \boldsymbol{J}_{k}, \\
\boldsymbol{\Sigma}_{k}(\boldsymbol{r})= & 2 C_{t}^{s} \boldsymbol{s}_{k}+2\left(C_{t}^{\Delta s}-C_{t}^{\nabla s}\right) \Delta \boldsymbol{s}_{k}-2 C_{t}^{\nabla s} \nabla \times\left(\nabla \times \boldsymbol{s}_{k}\right) \\
& +C_{t}^{T} \boldsymbol{T}_{k}+C_{t}^{F} \boldsymbol{F}_{k}+C_{t}^{\nabla j} \nabla \times \boldsymbol{j}_{k},
\end{aligned}
$$

$$
\begin{aligned}
& \boldsymbol{I}_{k}(\boldsymbol{r})=2 C_{t}^{j} \boldsymbol{j}_{k}+C_{t}^{\nabla j} \nabla \times \boldsymbol{s}_{k}, \\
& \mathrm{~B}_{k}(\boldsymbol{r})=2 C_{t}^{J 0} \boldsymbol{J}_{k} \delta-2 C_{t}^{J 1} \boldsymbol{\epsilon} \cdot \boldsymbol{J}_{k}+2 C_{t}^{J 2} \underline{\mathrm{J}}_{k}+C_{t}^{\nabla J} \boldsymbol{\epsilon} \cdot \nabla \rho_{k},
\end{aligned}
$$

$M_{k}(\boldsymbol{r})=C_{t}^{\tau} \rho_{k}$,

$\boldsymbol{C}_{k}(\boldsymbol{r})=C_{t}^{T} \boldsymbol{s}_{k}$

$\boldsymbol{D}_{k}(\boldsymbol{r})=C_{t}^{F} \boldsymbol{s}_{k}$.

All coupling constants $C_{t}$ in Eqs. (14) are taken with $t=0$ for $k=0$ (isoscalars), and with $t=1$ for $k=1,2,3$ (isovectors). ${ }^{1}$

The resulting HF equation can be written as a self-consistent eigenvalue problem,

$$
\int d^{3} \boldsymbol{r} \sum_{s t} \hat{h}\left(\boldsymbol{r}^{\prime} s^{\prime} t^{\prime}, \boldsymbol{r} s t\right) V_{k}^{*}(\boldsymbol{r} s t)=\varepsilon_{k} V_{k}^{*}\left(\boldsymbol{r}^{\prime} s^{\prime} t^{\prime}\right),
$$

which is solved by filling the lowest $A$ s.p. orbits in the density matrix (3).

\section{MODEL STUDY}

Recently, two computer codes capable of solving the selfconsistent equations for the isospin-invariant pnEDFs with the $p-n$ mixing, have been developed in parallel. The recent study [25] describes the scheme based on the code HFODD [26], which can treat symmetry-unrestricted nuclear shapes. In this work, we present the implementation based on the code HFBTHO, which assumes axial and time-reversal symmetries. The two codes complement each other in that HFODD is more general whereas HFBTHO is much faster, and thus they have different scopes and application ranges. While HFBTHO can employ the transformed oscillator basis that is particularly useful for weakly bound nuclei, the focus of the present work is to benchmark HFBTHO with HFODD; hence, we shall use the standard harmonic oscillator basis.

As in Ref. [25], we diagonalize the s.p. Routhian,

$$
\hat{h}^{\prime}=\hat{h}-\vec{\lambda} \circ \hat{\vec{t}}
$$

where $\hat{h}$ is given by Eq. (11) and contains kinetic, SkyrmepnEDF, and Coulomb-energy terms. The isocranking term [29], $-\vec{\lambda} \circ \hat{\vec{t}}$, depends on the isocranking frequency (isovector Fermi energy) $\vec{\lambda}$ and the s.p. isospin operator $\vec{t}=\hat{\vec{\tau}} / 2$.

For systems obeying the time-reversal symmetry, $\left\langle\hat{t}_{y}\right\rangle$ vanishes [17] and the rotation in isospace is described by a two-dimensional isocranking, that is,

$$
-\vec{\lambda} \circ \hat{\vec{t}}=-\lambda_{x} \hat{t}_{x}-\lambda_{z} \hat{t}_{z}
$$

The isocranking frequencies, $\lambda_{x}$ and $\lambda_{z}$, can be varied to control the isospin of the system. Following the methodology developed in Ref. [25], they are parametrized as

$$
\lambda_{z}=\lambda^{\prime} \cos \theta^{\prime}+\lambda_{\text {off }}, \quad \lambda_{x}=\lambda^{\prime} \sin \theta^{\prime},
$$

\footnotetext{
${ }^{1}$ Note that traditionally (cf. Ref. [17]) the same symbol $t$ denotes either the s.p. isospin coordinate $t= \pm \frac{1}{2}$ or isoscalar/isovector coupling constants $(t=0 / t=1)$. The meaning of $t$ thus has to be inferred from the context in which it is used.
} 
and the isocranking tilting angle $\theta^{\prime}$ is varied between $0^{\circ}$ and $180^{\circ}$, that is, the isovector Fermi energy on the $\lambda_{x}-\lambda_{z}$ plane moves along a shifted semicircle. In this work, numerical calculations were performed for the $A=78$ IASs with $T \simeq$ 11. We used the Skyrme EDF parametrization SkM* [30] and the s.p. basis space consisting of $N_{\mathrm{sh}}=16$ spherical harmonic-oscillator (HO) shells. Fore more details on the parameters employed, see Sec. IV.

In the absence of the Coulomb interaction, choosing $\lambda^{\prime}=21 \mathrm{MeV}, \lambda_{\text {off }}=0 \mathrm{MeV}$, and varying $\theta^{\prime}$ from $0^{\circ}$ to $180^{\circ}$ generates all the $A=78$ and $T=11$ IASs. The angles $\theta^{\prime}=0^{\circ}, 90^{\circ}$, and $180^{\circ}$ correspond to the HF solutions for ${ }^{78} \mathrm{Ni}\left(T_{z}=11\right),{ }^{78} \mathrm{Y}\left(T_{z}=0\right.$ in the odd-odd system $)$, and ${ }^{78} \mathrm{Sn}$ $\left(T_{z}=-11\right)$, respectively. Our example involves very exotic nuclei, including those beyond the proton drip line. We find this case interesting because the nuclei at both ends of the isobaric chain are heavy and doubly magic, thus spherical.

As discussed in Ref. [25], with the Coulomb term off, the value of $\lambda^{\prime}$ is roughly equal to the absolute value of difference between the proton and neutron Fermi energies in ${ }^{78} \mathrm{Ni}$ or ${ }^{78} \mathrm{Sn},\left|\lambda_{n}-\lambda_{p}\right|=21.18 \mathrm{MeV}$. Then, the isocranking term makes the Fermi energies of neutrons and protons almost equal. In the presence of the Coulomb interaction, however, a large asymmetry between $\left|\lambda_{n}-\lambda_{p}\right|$ develops between ${ }^{78} \mathrm{Ni}$ $(12.31 \mathrm{MeV})$ and ${ }^{78} \mathrm{Sn}(33.62 \mathrm{MeV})$. Therefore, to offset the difference of Fermi energies at $\theta^{\prime}=0^{\circ}$ and $180^{\circ}$ with Coulomb interaction present, we set the values

$$
\begin{aligned}
\lambda^{\prime} & =\frac{1}{2}\left\{\left|\lambda_{n}-\lambda_{p}\right|\left({ }^{78} \mathrm{Ni}\right)+\left|\lambda_{n}-\lambda_{p}\right|\left({ }^{78} \mathrm{Sn}\right)\right\}, \\
\lambda_{\text {off }} & =\frac{1}{2}\left\{\left|\lambda_{n}-\lambda_{p}\right|\left({ }^{78} \mathrm{Ni}\right)-\left|\lambda_{n}-\lambda_{p}\right|\left({ }^{78} \mathrm{Sn}\right)\right\} .
\end{aligned}
$$

Using these expressions, $\lambda^{\prime}=22.94 \mathrm{MeV}$ and $\lambda_{\text {off }}=$ $-10.92 \mathrm{MeV}$.

Figure 1 shows that, in the absence of the Coulomb interaction, the total energy is independent of $\theta^{\prime}$. This should be the case, as the pnEDF is isospin-invariant and thus the energy must be independent of the direction of the isospin vector. This also turned out to be an important test on the derived expressions and numerical code, as different terms of pnEDF

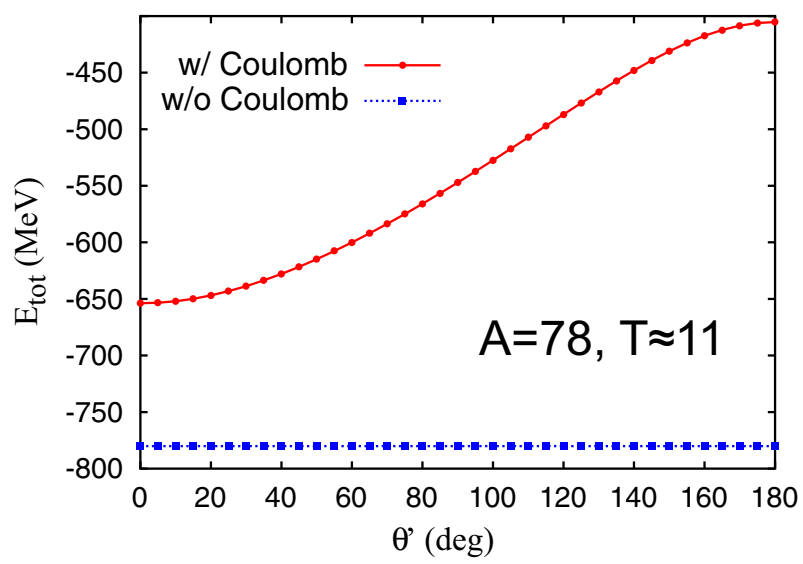

FIG. 1. (Color online) Total HF energy of the $A=78, T \approx 11$ IASs as a function of $\theta^{\prime}$ with (solid line) and without (dashed line) the isospin-symmetry-breaking Coulomb term.

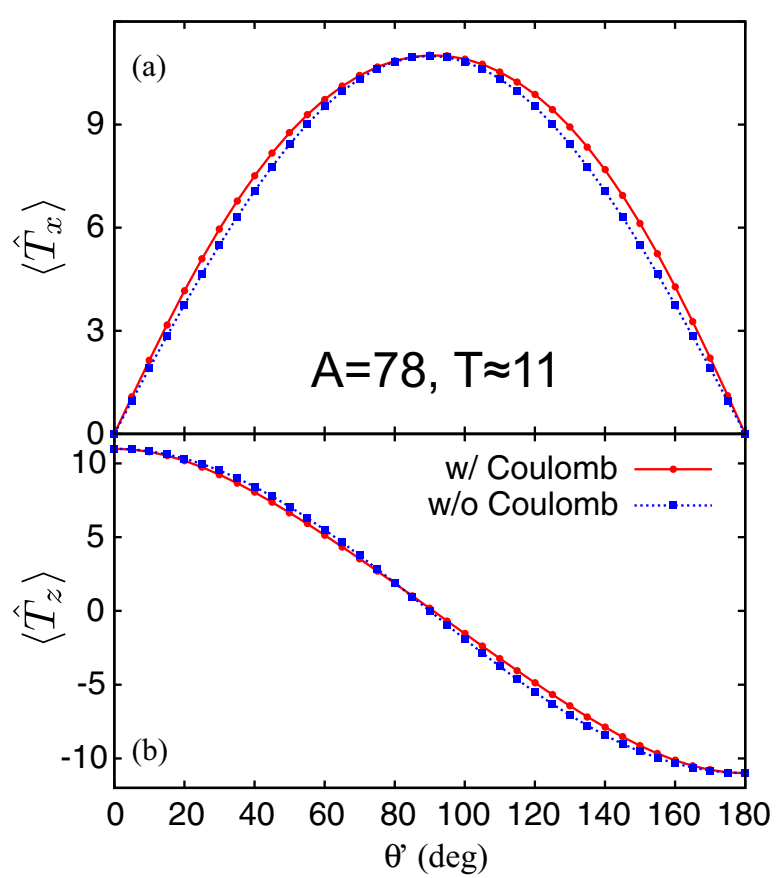

FIG. 2. (Color online) Similar as in Fig. 1, but for the expectation values of $\left\langle\hat{T}_{x}\right\rangle$ (a) and $\left\langle\hat{T}_{z}\right\rangle$ (b).

become active for different values of $\theta^{\prime}$. For $\theta^{\prime}=0^{\circ}$ and $180^{\circ}$, solutions are unmixed and the densities are block-diagonal in neutron and proton subspaces. At intermediate values of $\theta^{\prime}$, the solutions are $p-n$ mixed. For the special case of $\theta^{\prime}=90^{\circ}$, proton and neutron densities are equally mixed. When the Coulomb interaction is turned on, the total energy increases with $\theta^{\prime}$ (Fig. 1), because more and more protons replace neutrons and the Coulomb repulsion grows.

The degree of $p-n$ mixing can be directly inferred from the expectation values of $\left\langle\hat{T}_{x}\right\rangle$ plotted in Fig. 2(a). As expected, the $p$ - $n$ mixing increases with $\theta^{\prime}$ and reaches its maximum value for $\theta^{\prime}=90^{\circ}$, and then drops again. In Fig. 2(b), we show $\left\langle\hat{T}_{z}\right\rangle$ and it is seen that the values of $\theta^{\prime}=0^{\circ}, \sim 90^{\circ}$, and $180^{\circ}$ do correspond to ${ }^{78} \mathrm{Ni},{ }^{78} \mathrm{Y}$, and ${ }^{78} \mathrm{Sn}$, respectively. The behavior of $\left\langle\hat{T}_{z}\right\rangle$ and $\left\langle\hat{T}_{x}\right\rangle$ weakly depends on whether the Coulomb term is included or not. This is entirely due to our choice of the shifted semicircle (18), whereupon the linear constraint $\lambda_{\text {off }} \hat{t}_{z}$ absorbs the major part of the isovector component of the Coulomb interaction.

The Coulomb interaction breaks isospin and thus induces the isospin mixing in the HF wave function. To illustrate this, Fig. 3 shows the average value of $\left\langle\hat{T}^{2}\right\rangle$ for the converged HF solutions. For the considered case of the $T=11$ systems, $\left\langle\hat{T}^{2}\right\rangle$ should be exactly equal to $11 \times 12=132$ in the absence of isospin mixing. However, as shown in Fig. 3, even in the absence of the Coulomb interaction, $\left\langle\hat{T}^{2}\right\rangle$ slightly deviates from this value. At the origin of this effect is the spurious isospin mixing [31-33]. Indeed, within the mean-field approximation, the isospin symmetry is broken spontaneously as the HF wave function is not an isospin eigenstate. However, since the Skyrme EDF is isospin covariant [15,34], the HF solutions corresponding to different orientations in the isospin space are degenerate in energy. While the neutron-proton 


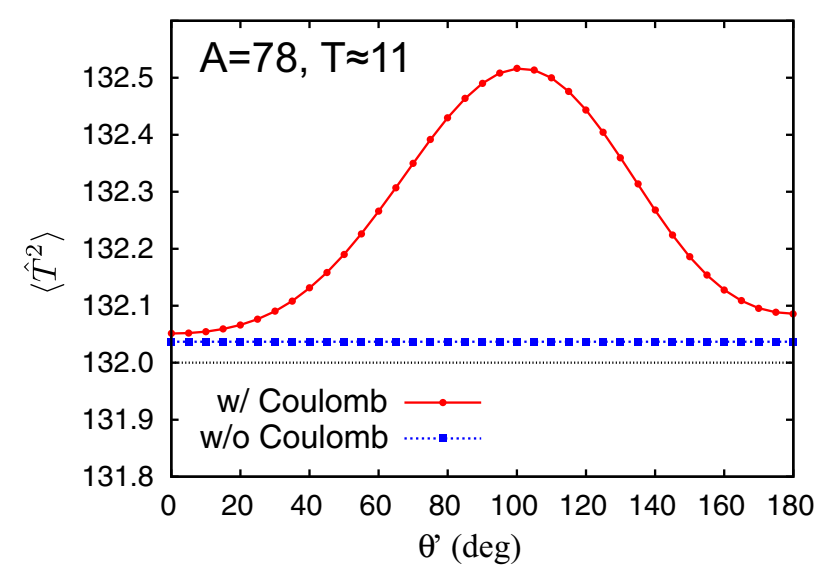

FIG. 3. (Color online) Similar as in Fig. 1, but for $\left\langle\hat{T}^{2}\right\rangle$.

mixing changes with the angle $\theta,\left\langle\hat{T}^{2}\right\rangle$ must remain the same in the absence of the Coulomb interaction. In the presence of the Coulomb term, the isospin mixing is very small in the isospin-stretched $\left|T_{z}\right|=11$ configurations (for $\theta^{\prime}=0^{\circ}$ and $180^{\circ}$ ) and reaches its maximum around $\theta^{\prime}=90^{\circ}$ for $T_{z}=0$ $[33,35]$.

The s.p. Routhians as functions of $\theta^{\prime}$ are shown in Figs. 4 (without Coulomb) and 5 (with Coulomb). Eleven spherical neutron levels and seven proton levels are occupied at $\theta^{\prime}=0^{\circ}$, and the neutron and proton Fermi energies are shifted in such a way that the gaps in the s.p. spectra appear at $A=78$ around $-15 \mathrm{MeV}$ (Fig. 4) and $-10 \mathrm{MeV}$ (Fig. 5). Our choice of $\vec{\lambda}$ guarantees that, in the presence of the Coulomb interaction, the s.p. Routhians near the Fermi surface do not cross as functions of $\theta^{\prime}$; this would have caused a drastic structural changes of the

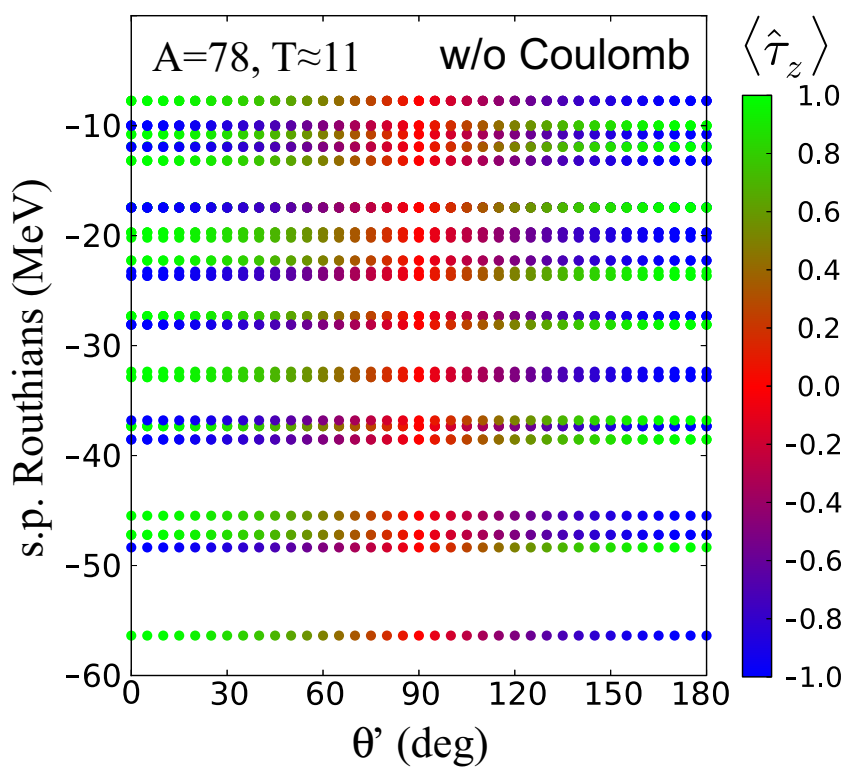

FIG. 4. (Color online) Single-particle Routhians as functions of $\theta^{\prime}$ for $T \approx 11$ configurations in the $A=78$ systems. The Coulomb interaction is not included. Points are colored according to the s.p. expectation values of $\left\langle\hat{\tau}_{z}\right\rangle$. At $\theta^{\prime}=0^{\circ}$, neutron and proton states are plotted up to $1 g_{7 / 2}$ and $2 p_{1 / 2}$, respectively.

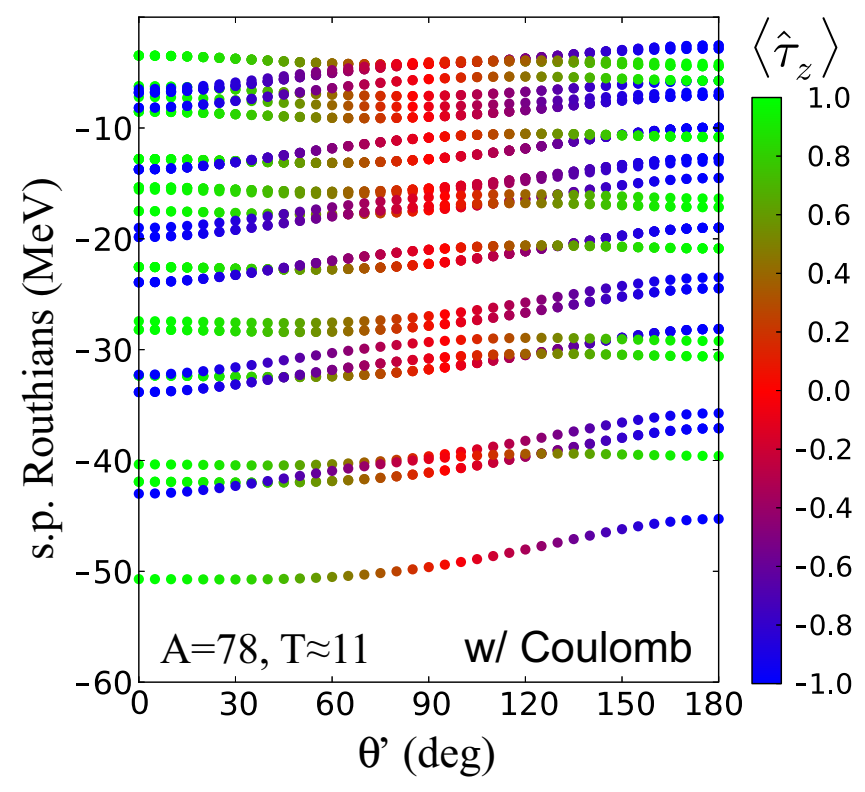

FIG. 5. (Color online) Similar to Fig. 4, but with the Coulomb interaction included.

mean field and made the adiabatic tracing of the $T \simeq 11$ IAS as a function of $\theta^{\prime}$ extremely difficult. At $\theta^{\prime}=0^{\circ}$ and $180^{\circ}$, the s.p. states have pure values of $\left\langle\hat{t}_{z}\right\rangle= \pm \frac{1}{2}$. At $\theta^{\prime} \sim 90^{\circ}$, most of the s.p. Routhians have $\left\langle\hat{t}_{z}\right\rangle$ close to zero, that is, they are fully $p-n$ mixed.

Figure 6 displays s.p. HF energies, that is, s.p. Routhians with the isocranking term removed. Note that these are not eigenvalues but the average values of the HF Hamiltonian, calculated for states that are eigenstates of the Routhian (16). With increasing $\theta^{\prime}$, owing to the increasing Coulomb field,

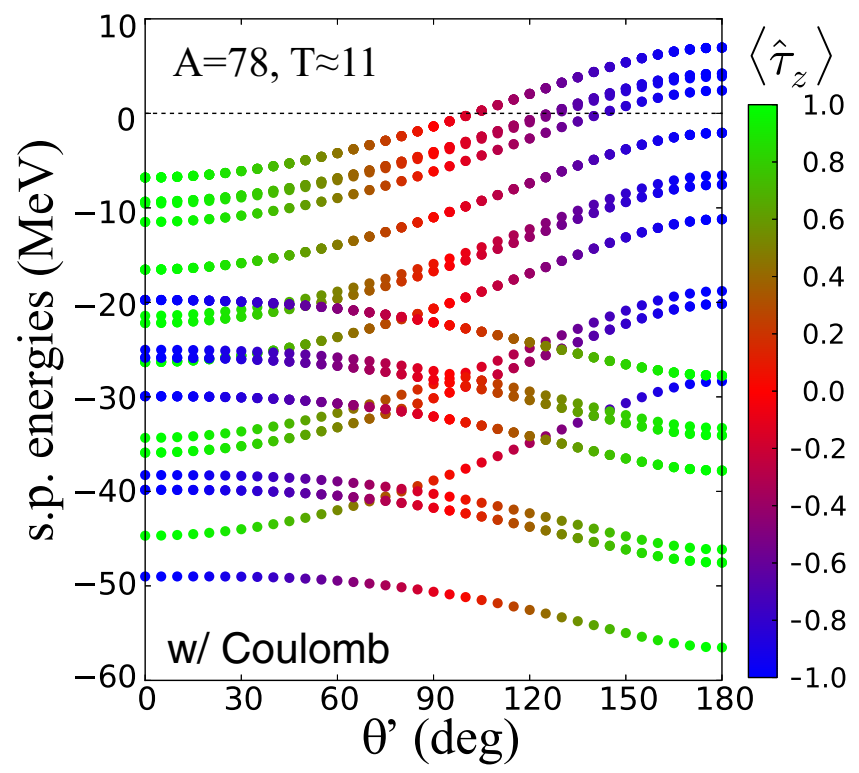

FIG. 6. (Color online) Similar to Fig. 4 but for the s.p. energies with the Coulomb interaction included. Only the occupied states are plotted; that is, at $\theta^{\prime}=0^{\circ}$, neutron and proton states are plotted up to $1 g_{9 / 2}$ and $1 f_{7 / 2}$ shells, respectively. 


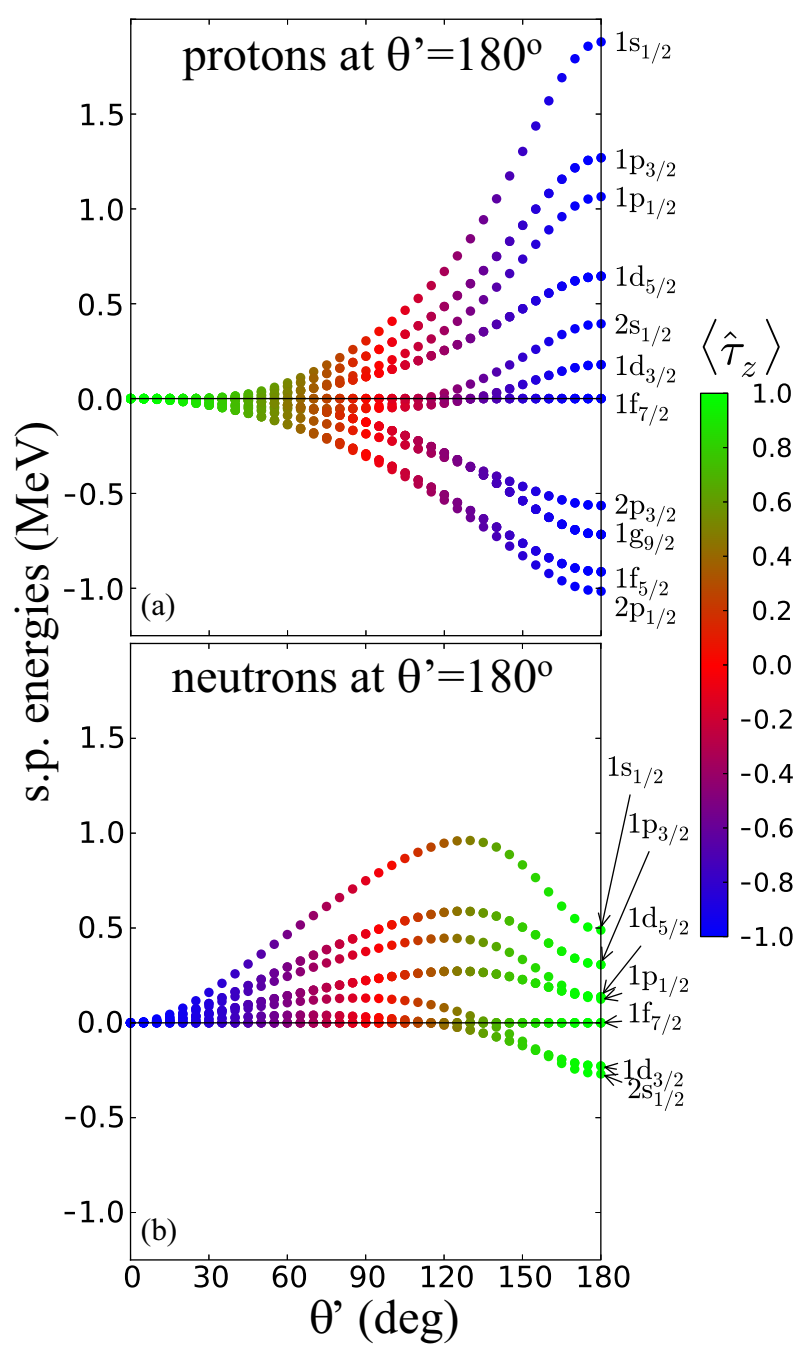

FIG. 7. (Color online) Evolution of s.p. energies of occupied HF states (with Coulomb) originating from proton (top) and neutron (bottom) shells at $\theta^{\prime}=180$ with respect to the energy of the $1 f_{7 / 2}$ shell, and relative to the corresponding values at $\theta^{\prime}=0^{\circ}$. That is, the energies plotted are $\left[\varepsilon_{i}\left(\theta^{\prime}\right)-\varepsilon_{f_{7 / 2}}\left(\theta^{\prime}\right)\right]-\left[\varepsilon_{i}\left(0^{\circ}\right)-\varepsilon_{f_{7 / 2}}\left(0^{\circ}\right)\right]$.

s.p. states that increase proton (neutron) component gradually increase (decrease) in energy.

To better visualize the relative shifts of s.p. levels with $\theta^{\prime}$, in Fig. 7 we show s.p. energies relative to the energy of the $1 f_{7 / 2}$ shell. The figure nicely illustrates the effect of the Coulomb interaction on the proton components of the s.p. orbits: the relative level shifts correlate with their binding energies and $\ell$ values [36,37]. Indeed, the deeply (loosely) bound levels, which have smaller (larger) rms radii and thus experience stronger (weaker) Coulomb repulsion, are shifted up (down) in energy relative to the high- $\ell 1 f_{7 / 2}$ shell.

Some of the calculated $A=78, T \simeq 11$ IASs are predicted to appear beyond the proton drip line. As seen in Fig. 6, energy of the $1 g_{9 / 2}$ level (which is neutron at $\theta^{\prime}=0^{\circ}$ and proton at $\theta^{\prime}=180^{\circ}$ ) becomes positive at around $\theta^{\prime}=100^{\circ}$, where $\left\langle\hat{T}_{z}\right\rangle \approx-1.5$. At $\theta^{\prime}=180^{\circ}$, energies of the $1 g_{9 / 2}, 1 f_{5 / 2}, 2 p_{1 / 2}$, and $2 p_{3 / 2}$ shells are positive. However, all these states are well localized by the Coulomb barrier, and thus correspond

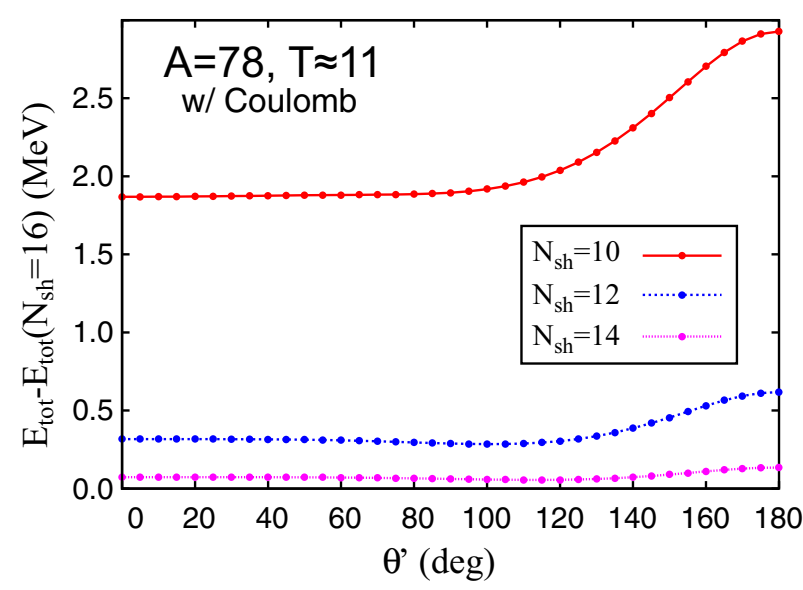

FIG. 8. (Color online) The total HF energy (with Coulomb) for $A=78, T \approx 11$ calculated with $N_{\mathrm{sh}}=10,12$, and $14 \mathrm{HO}$ shells relative to that with $N_{\mathrm{sh}}=16$ shells as a function of $\theta^{\prime}$.

to narrow resonances, whose energies can be reasonably well described within the HO basis expansion [37].

In Fig. 8, we show the convergence of the total HF energy with respect to the total number of $\mathrm{HO}$ shells $N_{\mathrm{sh}}$. Calculations using $N_{\text {sh }}=14$ are not yet completely converged, with the energy difference between $N_{\mathrm{sh}}=16$ and $N_{\mathrm{sh}}=14$ varying around $73 \mathrm{keV}$ at $\theta^{\prime}=0^{\circ}$ and $135 \mathrm{keV}$ at $\theta^{\prime}=180^{\circ}$. Although it is expected that by increasing $N_{\text {sh }}$ one may still lower the energy, the change is expected to be less than $100 \mathrm{keV}$, and the results presented in this study are not expected to change significantly. A delayed convergence beyond $\theta^{\prime}=140^{\circ}$ shows that the higher $\mathrm{HO}$ shells are more important at $\theta^{\prime}=180^{\circ}$ than at $\theta^{\prime}=0^{\circ}$; that is, a larger model space is required for the description of the unbound proton resonances [38]. Nevertheless, as seen in Fig. 9, even near $\theta^{\prime}=180^{\circ}$ no sudden increase of proton rms radii is obtained.

To investigate properties of the unbound proton orbits, for the ground states of $A=78, T \simeq\left|T_{z}\right|$ nuclei, we performed the HFBRAD [39] calculations (without $p-n$ mixing). In Fig. 10, we show results obtained for the $1 g_{9 / 2}$ and $2 p_{1 / 2}$ proton states. For each $A=78$ isobar, a dot is placed at the values of s.p. energies and radii, and lines show standard total effective HF proton potentials. The total effective HF proton potential consists of the standard central, spin-orbit, centrifugal, and Coulomb terms. The proton $1 g_{9 / 2}$ orbit in ${ }^{78} \mathrm{Zr}$ is bound, and in ${ }^{78} \mathrm{Mo}$ it becomes slightly unbound. This result is consistent with the experimental observation, whereby the last bound nucleus of the $A=78$ isobaric chain, which is experimentally known, is ${ }^{78} \mathrm{Zr}\left(T_{z}=-1\right)$. The $\mathrm{rms}$ radii of the proton $1 g_{9 / 2}$ orbits are about $5 \mathrm{fm}$, and the s.p. wave functions are still localized, even if the orbits become unbound. This is because the $1 g_{9 / 2}$ and $2 p_{1 / 2}$ protons occupy states well below the potential barrier, which pushes the proton continuum up in energy, thus effectively extending the range of nuclear landscape into the proton-unstable region $[21,40]$.

It is worth noting that the $2 p_{1 / 2}$ orbit, which has a small centrifugal barrier, is bound up to around $\left(\theta^{\prime}=125^{\circ},\left\langle\hat{T}_{z}\right\rangle \approx\right.$ $-5.7)$ in Fig. 6. This is consistent with Fig. 10 that shows that the s.p. energy of $2 p_{1 / 2}$ is unbound in ${ }^{78} \mathrm{Ru}\left(\left\langle\hat{T}_{z}\right\rangle=-5\right)$. 


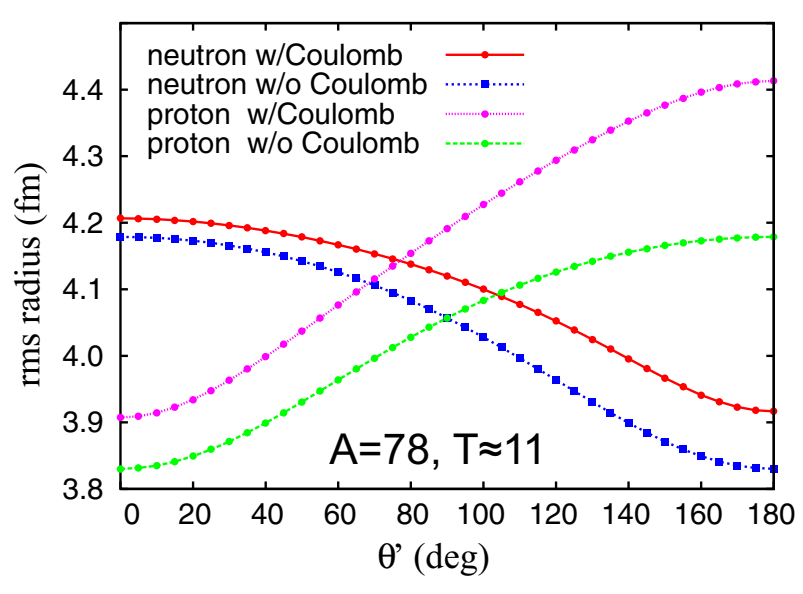

FIG. 9. (Color online) Neutron and proton rms radii as functions of $\theta^{\prime}$.

In the presence of $p-n$ mixing, the proton components of the s.p. states are smaller than those in the pure proton states, and this effectively reduces the repulsive Coulomb energies of the $1 g_{9 / 2}$ orbits.

\section{BENCHMARKING WITH HFODD}

To demonstrate that the isospin-invariant formalism has been properly implemented, we provide a detailed comparison between the HFODD [25] and HFBTHO frameworks. This benchmarking is meaningful as the two pnEDF codes were developed independently and have fairly different structures.
In particular, the HF equations in HFODD [26] are solved in three-dimensional Cartesian basis while HFBTHO employs the two-dimensional cylindrical basis.

Calculations were performed for the $A=40, T \simeq 8 \mathrm{de}$ formed IASs with SkM* EDF parametrization using the s.p. basis space of $N_{\mathrm{sh}}=10$. The oscillator length was assumed to be $b=1.697626 \mathrm{fm}$. The mass constant in Eq. (5) was fixed at $\hbar^{2} / 2 m=20.73 \mathrm{MeV} \mathrm{\textrm {fm } ^ { 2 }}$. As far as integration is concerned, we used $N_{\mathrm{GH}}=26$ Gauss-Hermite nodes for each Cartesian coordinate in HFODD, whereas in HFBTHO the numbers of Gauss-Hermite ( $\rho$-direction) and Gauss-Laguerre (z-direction) nodes were assumed to be equal: $N_{\mathrm{GH}}=N_{\mathrm{GL}}=$ 40. In addition, in HFBTHO, the number of Gauss-Legendre nodes used in the integration of the direct Coulomb field was set to 80 , and the Coulomb length scale was taken to be $L=50 \mathrm{fm}$. This set of parameters was recommended as a default value in the latest version of the HFBTHO, as it provides a sufficient precision on the direct Coulomb energy [23]. Without Coulomb, the isocranking frequency was set to $\lambda^{\prime}=27.092394 \mathrm{MeV}$ and $\lambda_{\text {off }}=0 \mathrm{MeV}$. With Coulomb, we took the values $\lambda^{\prime}=28.613615 \mathrm{MeV}$ and $\lambda_{\text {off }}=$ $-6.010741 \mathrm{MeV}$.

The benchmarking results for the deformed case are shown in Tables I and II for $\theta^{\prime}=0^{\circ}$ and $90^{\circ}$, respectively. In the absence of the Coulomb term, the difference in the total energy $E_{\text {tot }}$ is less than $20 \mathrm{eV}$, and the total isospin $\left\langle\hat{T}^{2}\right\rangle$ agrees up to the sixth decimal place. With the inclusion of the Coulomb term, the agreement is slightly reduced but is still excellent. A comparison between HFBTHO and HFODD was also performed for the spherical $A=48, T \simeq 4$ IASs, and the results are
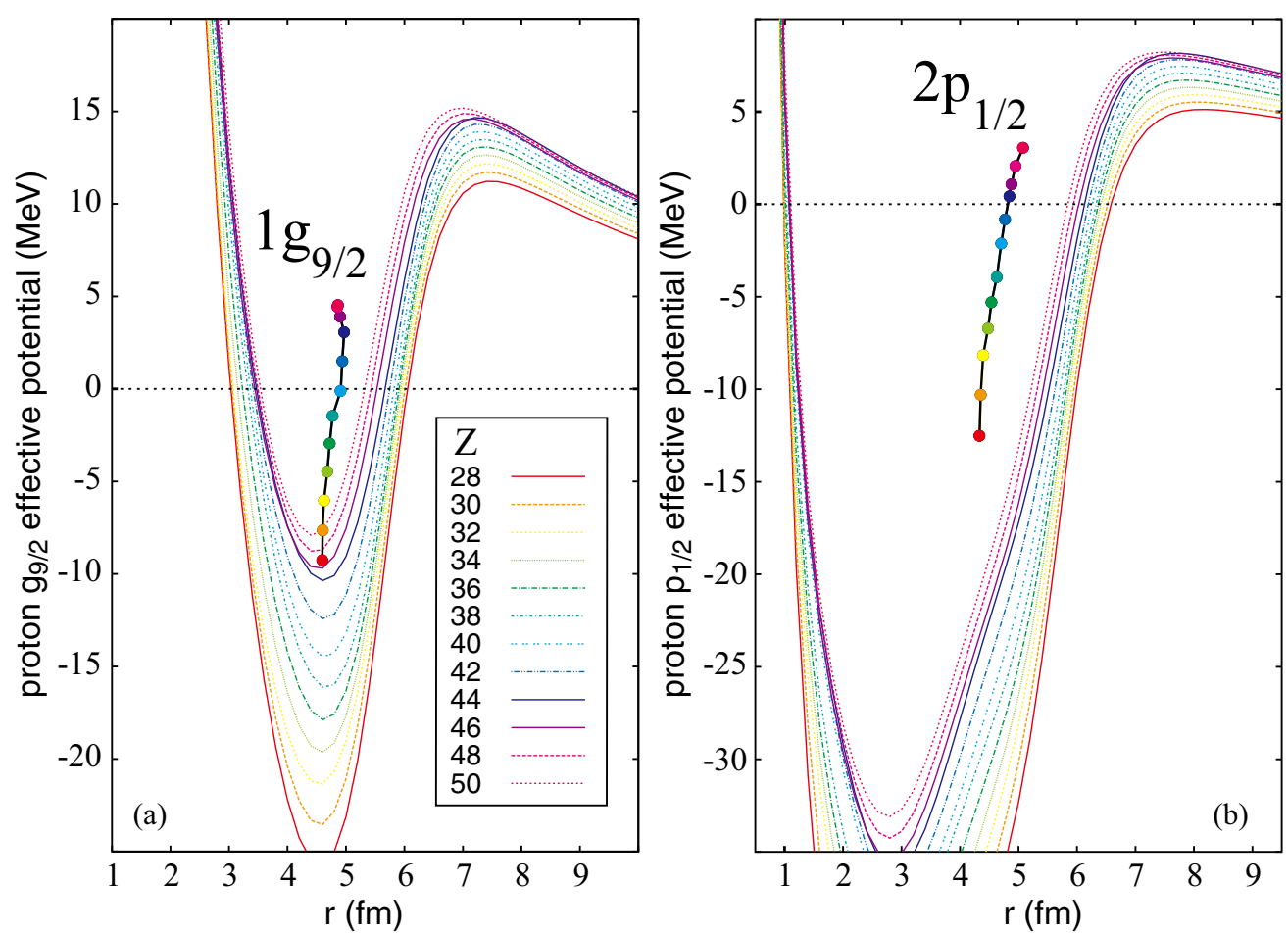

FIG. 10. (Color online) Lines: proton effective HF potential (with Coulomb and centrifugal terms included), calculated for the $A=78$, $T \simeq\left|T_{z}\right|$ isobars for $\ell=4$ (a) and $\ell=1$ (b). Dots: rms radii and s.p. energies of the proton $1 g_{9 / 2}$ (a) and $1 p_{1 / 2}$ states. 
TABLE I. Benchmarking of HFODD with HFBTHO for the deformed $A=40, T \simeq 8 \mathrm{IASs}$ using $\operatorname{SkM}^{*} \operatorname{EDF}\left(N_{\mathrm{sh}}=10\right)$, and $\theta^{\prime}=$ $0^{\circ}\left({ }^{40} \mathrm{Mg}\right)$. Other parameters are $\lambda^{\prime}=27.092394 \mathrm{MeV}, \lambda_{\text {off }}=0 \mathrm{MeV}$ (without Coulomb), and $\lambda^{\prime}=28.613615 \mathrm{MeV}, \lambda_{\text {off }}=-6.010741$ $\mathrm{MeV}$ (with Coulomb). Shown are various contributions to the total binding energy $E_{\text {tot }}$ (in $\mathrm{MeV}$ ), rms radii (in fm), expectation values of $T^{2}, T_{z}$, and $T_{x}$, and the quadrupole deformation $\beta_{2}$. The digits which do not coincide in HFODD and HFBTHO are marked in bold.

\begin{tabular}{|c|c|c|c|c|}
\hline & \multicolumn{2}{|c|}{ Without Coulomb } & \multicolumn{2}{|c|}{ With Coulomb } \\
\hline & HFODD & НFBTHO & HFODD & HFBTHO \\
\hline$E_{\text {tot }}$ & -303.42519 & -303.42520 & -276.47641 & -276.47643 \\
\hline$E_{\text {kin }}^{(\mathrm{n})}$ & 498.448466 & 498.448464 & 495.53929 & 495.53930 \\
\hline$E_{\text {kin }}^{(\mathrm{p})}$ & 175.371764 & 175.371762 & 171.30205 & 171.30206 \\
\hline$E_{\text {pot }}$ & -977.24542 & -977.24543 & -970.09923 & -970.09926 \\
\hline$E_{\mathrm{SO}}$ & -34.357903 & -34.357905 & -33.184812 & -33.184816 \\
\hline$E_{\text {Cou }}^{(\mathrm{dir})}$ & & & 30.920704 & 30.920697 \\
\hline$E_{\mathrm{Cou}}^{(\mathrm{exc})}$ & & & -4.139228 & -4.139228 \\
\hline$r_{\mathrm{rms}}^{(\mathrm{n})}$ & 3.697718 & 3.697718 & 3.709975 & 3.709975 \\
\hline$r_{\text {rms }}^{(\mathrm{p})}$ & 3.176356 & 3.176356 & 3.217587 & 3.217587 \\
\hline$T^{2}$ & 72.022743 & 72.022743 & 72.023123 & 72.023123 \\
\hline$T_{z}$ & 8.000000 & 8.000000 & 8.000000 & 8.000000 \\
\hline$T_{x}$ & 0.000000 & 0.000000 & 0.000000 & 0.000000 \\
\hline$\beta_{2}$ & 0.304201 & 0.304201 & 0.311518 & 0.311518 \\
\hline
\end{tabular}

presented in Table III for $\theta^{\prime}=90^{\circ}$ where the differences between the two codes are largest. In this case, it is found that deviation in the total energy is about $20 \mathrm{eV}$.

It is to be noted that both HFODD and HFBTHO use the same number of basis harmonic oscillator states. Moreover, as it has been demonstrated previously [23], using a sufficient number of quadrature points in HFBTHO and HFODD, the results of both solvers agree with a high accuracy of several $\mathrm{eV}$. The differences between the two codes with the Coulomb potential turned on can be traced back to different techniques used to compute the direct Coulomb field: the solver HFODD uses a more accurate Green's function approach. The benchmark

TABLE II. Similar to Table I but for $\theta^{\prime}=90^{\circ}\left({ }^{40} \mathrm{Ca}\right)$.

\begin{tabular}{|c|c|c|c|c|}
\hline & \multicolumn{2}{|c|}{ Without Coulomb } & \multicolumn{2}{|c|}{ With Coulomb } \\
\hline & HFODD & НFBTHO & HFODD & HFBTHO \\
\hline$E_{\text {tot }}$ & -303.42519 & -303.42520 & -234.429 & -234.419 \\
\hline$E_{\mathrm{kin}}^{(\mathrm{n})}$ & 336.910115 & 336.910113 & 333.68 & 333.71 \\
\hline$E_{\mathrm{kin}}^{(\mathrm{p})}$ & 336.910115 & 336.910113 & 318.704 & 318.713 \\
\hline$E_{\mathrm{pot}}^{\mathrm{nit}}$ & -977.245420 & -977.24543 & -954.79 & -954.83 \\
\hline$E_{\mathrm{SO}}$ & -34.357903 & -34.357905 & -31.49 & -31.51 \\
\hline$E_{\mathrm{Cou}}^{(\mathrm{dir})}$ & & & 75.105 & 75.106 \\
\hline$E_{\mathrm{Cou}}^{(\mathrm{exc})}$ & & & -7.12522 & -7.12525 \\
\hline$r_{\text {rms }}^{(\mathrm{n})}$ & 3.549360 & 3.549360 & 3.5930 & 3.5928 \\
\hline$r_{\mathrm{rms}}^{(\mathrm{p})}$ & 3.549360 & 3.549360 & 3.63519 & 3.63512 \\
\hline$T^{2}$ & 72.022743 & 72.022743 & 72.149 & 72.155 \\
\hline$T_{z}$ & 0.000000 & 0.000000 & 0.15649 & 0.15652 \\
\hline$T_{x}$ & 8.000000 & 8.000000 & 8.0051 & 8.0054 \\
\hline$\beta_{2}$ & 0.304201 & 0.304201 & 0.3184 & 0.3182 \\
\hline
\end{tabular}

TABLE III. Similar to Table I but for the spherical $A=48, T \simeq 4$ IASs and $\theta^{\prime}=90^{\circ}\left({ }^{48} \mathrm{Cr}\right)$. Other parameters are: $\lambda^{\prime}=11.0 \mathrm{MeV}$, $\lambda_{\text {off }}=0 \mathrm{MeV}$ (without Coulomb), and $\lambda^{\prime}=12.0 \mathrm{MeV}, \lambda_{\text {off }}=-8.0$ $\mathrm{MeV}$ (with Coulomb).

\begin{tabular}{|c|c|c|c|c|}
\hline & \multicolumn{2}{|c|}{ Without Coulomb } & \multicolumn{2}{|c|}{ With Coulomb } \\
\hline & HFODD & HFBTHO & HFODD & HFBTHO \\
\hline$E_{\text {tot }}$ & -491.243706 & -491.243724 & -389.8454 & -389.8438 \\
\hline$E_{\text {kin }}^{(\mathrm{n})}$ & 422.93152 & 422.93158 & 415.69 & 415.71 \\
\hline$E_{\text {kin }}^{(\mathrm{p})}$ & 422.93152 & 422.93143 & 404.673 & 404.679 \\
\hline$E_{\mathrm{pot}}$ & -1337.10675 & -1337.10673 & -1310.44 & -1310.46 \\
\hline$E_{\mathrm{SO}}$ & -36.736418 & -36.736417 & -34.1216 & -34.1240 \\
\hline$E_{\mathrm{Cou}}^{(\mathrm{dir})}$ & & & 109.3774 & 109.3781 \\
\hline$E_{\mathrm{Cou}}^{\text {(exc) }}$ & & & -9.14576 & -9.14581 \\
\hline$r_{\text {rms }}^{(\mathrm{n})}$ & 3.497939 & 3.497940 & 3.52633 & 3.52628 \\
\hline$r_{\text {rms }}^{(\mathrm{p})}$ & 3.497939 & 3.497939 & 3.57787 & 3.57784 \\
\hline$T^{2}$ & 20.037818 & 20.037818 & 20.0756 & 20.0771 \\
\hline$T_{z}$ & 0.000000 & 0.000003 & -0.012676 & -0.012663 \\
\hline$T_{x}$ & 4.000000 & 4.000000 & 4.00264 & 4.00278 \\
\hline
\end{tabular}

examples discussed in this section demonstrate that the $p$ - $n$ mixing has been implemented correctly in both codes. Presently, we are in process of implementing the cylindrical Green's function treatment of the Coulomb potential into HFBTHO, and it is expected that the agreement between the two codes will further improve.

\section{SUMMARY AND OUTLOOK}

The description of weakly bound complex nuclei is a demanding task, as it requires the understanding and control of three crucial aspects of the nuclear many-body problem: interaction, correlations, and coupling to the low-lying particle continuum. Here, the theoretical tool of choice is nuclear density functional theory based on the self-consistent EDF approach. The quest for a truly universal nuclear EDF is one of the main themes of theoretical nuclear structure research today.

The isospin channel of the nuclear EDF still remains largely unexplored. In the existing functionals, only isoscalar and $t_{z}$ components of the isovector densities are used as building blocks. In a completely isospin-invariant formalism, all three isovector density components should be considered: those correspond to $p-n$ mixed densities. For heavy nuclei, possessing significant neutron excess, the omission of $p-n$ mixed densities can be justified as neutron and protons occupy different shells. However, for lighter systems, neutrons and protons usually occupy the same shell-model orbits, and $p-n$ mixed densities are likely to appear. Some limited experimental evidence suggests that the $p$ - $n$ fields play a role near the $N=Z$ line.

In the present work, we have developed a new Skyrme-EDF approach with the inclusion of $p-n$ mixed densities [17]. The expressions for the densities and HF fields have been worked out in the axial limit. The present 2D HFBTHO implementation that includes mixed $p-n$ densities and fields is fairly fast, and this allows for systematic large-scale surveys. The new 
framework has been tested in the HF limit and it was benchmarked with 3D HFODD spherical and deformed calculations [25]. The basic features of the $p-n$ mixed HF formalism have been investigated by studying the $A=78, T \approx 11$ IAS chain. In particular, we investigated the isospin breaking effects and stability of solutions obtained for the proton-unbound systems.

The present work has been primarily devoted to the detailed test of the newly developed isospin-invariant density functional formalism. In the near future, we intend to perform realistic HFB calculations by including the generalized pairing interaction in both isoscalar and isovector channels in order to study the importance of the $T=0$ pairing densities and fields on the structure of nuclei close to the $N=Z$ line and the impact of $p-n$ mixing on $\beta$ decays.

\section{ACKNOWLEDGMENTS}

We would like to express our deep gratitude to our friend and colleague, Mario Stoitsov, for his contributions in the initial stages of this work. J.A.S. would like to acknowledge I. Maqbool and P. A. Ganai for discussions. This work was supported by the U.S. Department of Energy under Contracts No. DE-FG02-96ER40963 (University of Tennessee), No. DESC0008499 (NUCLEI SciDAC Collaboration), and No. DEFG02-06ER41407 (JUSTIPEN, Japan-U.S. Theory Institute for Physics with Exotic Nuclei); by the Academy of Finland and University of Jyväskylä within the FIDIPRO program; by the Polish National Science Center under Contract No. 2012/07/B/ST2/03907; and by JSPS KAKENHI (Grants No. 20105003, No. 24105006, No. 25287065, and No. 25287066). Computational resources were provided through an INCITE award "Computational Nuclear Structure" by the National Center for Computational Sciences (NCCS) and the National Institute for Computational Sciences (NICS) at Oak Ridge National Laboratory. A part of the numerical calculations were also carried out on SR16000 at the Yukawa Institute for Theoretical Physics in Kyoto University and on the RIKEN Integrated Cluster of Clusters (RICC) facility.

\section{APPENDIX: CYLINDRICAL SYMMETRY}

In the case of cylindrical symmetry, the third component $J_{z}$ of the total angular-momentum is conserved and provides a good quantum number $\Omega_{k}$. The HF s.p. wave functions in the axial limit can be written as [22]

$$
\begin{aligned}
V_{k}(\boldsymbol{r} s t)= & V_{k}^{+}(r z t) e^{i \Lambda^{-} \phi} \chi_{+1 / 2}(s) \\
& +V_{k}^{-}(r z t) e^{i \Lambda^{+} \phi} \chi_{-1 / 2}(s),
\end{aligned}
$$

where $\Lambda^{ \pm}=\Omega_{k} \pm 1 / 2$ and $(r, \phi, z)$ are the cylindrical coordinates defining the three-dimensional position vector, $r=$ $(r \cos \phi, r \sin \phi, z)$ and $z$ is the chosen symmetry axis. In the following section, the expressions in the cylindrical coordinate basis under the axial symmetry are provided for the local particle-hole densities. In the second section, expressions of the derivatives of the local densities, which are used in the evaluation of the HF potentials, are given.

\section{Particle-hole densities}

We give the expression for particle density $\rho$, kinetic density $\tau$, spin density $\boldsymbol{s}$, spin-kinetic density $\boldsymbol{T}$, current density $\boldsymbol{j}$, and spin-current density J. Tensor-kinetic density $\boldsymbol{F}$ is not used and its expression is omitted.

(a) Scalar particle density

$$
\rho_{m}(\boldsymbol{r})=\left.\rho_{m}\left(\boldsymbol{r}, \boldsymbol{r}^{\prime}\right)\right|_{\boldsymbol{r}=\boldsymbol{r}^{\prime}}
$$

where $m$ takes values $0,1,2$, and 3. Suffix 0 represents isoscalar component of the density and 1 to 3 are the isovector components. Expressing the density in terms of HFB wave functions (A1), we obtain

$$
\rho_{m}(\boldsymbol{r})=\sum_{t t^{\prime}} \rho^{t t^{\prime}}(r z) \tau_{t^{\prime} t}^{m}
$$

where

$$
\rho^{t t^{\prime}}(r z)=\sum_{k}\left[V_{k t}^{+*} V_{k t^{\prime}}^{+}+V_{k t}^{-*} V_{k t^{\prime}}^{-}\right],
$$

and we use the abbreviation of HFB wave functions

$$
V_{k t}^{ \pm} \equiv V_{k}^{ \pm}(r z t)
$$

Isospin components of the particle density are given by

$$
\begin{aligned}
\rho_{0}(\boldsymbol{r}) & =\rho^{n n}(r z)+\rho^{p p}(r z) \\
& =\frac{1}{2}\left(\rho^{n n}(r z)+\rho^{p p}(r z)+\text { c.c. }\right), \\
\rho_{1}(\boldsymbol{r}) & =\rho^{n p}(r z)+\rho^{p n}(r z)=\rho^{n p}(r z)+\text { c.c. }, \\
\rho_{2}(\boldsymbol{r}) & =i\left[\rho^{n p}(r z)-\rho^{p n}(r z)\right]=i \rho^{n p}(r z)+\text { c.c. }, \\
\rho_{3}(\boldsymbol{r}) & =\rho^{n n}(r z)-\rho^{p p}(r z) \\
& =\frac{1}{2}\left(\rho^{n n}(r z)-\rho^{p p}(r z)+\text { c.c. }\right) .
\end{aligned}
$$

The isospin structure of the particle density given by Eqs. (A6)-(A9) is identical for all the following particle-hole densities, and these expressions shall not be repeated in the following.

(b) Kinetic density

$$
\tau_{m}(\boldsymbol{r})=\left.\left(\nabla \cdot \nabla^{\prime}\right) \rho_{m}\left(\boldsymbol{r}, \boldsymbol{r}^{\prime}\right)\right|_{\boldsymbol{r}=\boldsymbol{r}^{\prime}}=\sum_{t t^{\prime}} \tau^{t t^{\prime}}(\boldsymbol{r} z) \tau_{t^{\prime} t}^{m},
$$

where

$$
\begin{aligned}
\tau^{t t^{\prime}}(r z)= & \sum_{k}\left\{\left[\partial_{r} V_{k t}^{+*}\right]\left[\partial_{r} V_{k t^{\prime}}^{+}\right]+\left[\partial_{r} V_{k t}^{-*}\right]\left[\partial_{r} V_{k t^{\prime}}^{-}\right]\right. \\
& +\frac{\left(\Lambda^{-}\right)^{2}}{r^{2}} V_{k t}^{+*} V_{k t^{\prime}}^{+}+\frac{\left(\Lambda^{+}\right)^{2}}{r^{2}} V_{k t}^{-*} V_{k t^{\prime}}^{-} \\
& \left.+\left[\partial_{z} V_{k t}^{+*}\right]\left[\partial_{z} V_{k t^{\prime}}^{+}\right]+\left[\partial_{z} V_{k t}^{-*}\right]\left[\partial_{z} V_{k t^{\prime}}^{-}\right]\right\} .
\end{aligned}
$$

(c) Pseudovector spin density

$$
\boldsymbol{s}_{m}(\boldsymbol{r})=\left.\boldsymbol{s}_{m}\left(\boldsymbol{r}, \boldsymbol{r}^{\prime}\right)\right|_{\boldsymbol{r}=\boldsymbol{r}^{\prime}}=\sum_{t t^{\prime}} \boldsymbol{s}^{t t^{\prime}}(r z) \tau_{t^{\prime} t}^{m},
$$


where

$$
\begin{aligned}
\boldsymbol{s}^{t t^{\prime}}(r z)= & \sum_{k}\left[\boldsymbol{e}_{r} s_{r}^{t t^{\prime}}(r z)+\boldsymbol{e}_{\phi} s_{\phi}^{t t^{\prime}}(r z)+\boldsymbol{e}_{z} s_{z}^{t t^{\prime}}(r z)\right] \\
= & \sum_{k}\left\{\boldsymbol{e}_{r}\left[V_{k t}^{-*} V_{k t^{\prime}}^{+}+V_{k t}^{+*} V_{k t^{\prime}}^{-}\right]\right. \\
& +\boldsymbol{e}_{\phi} i\left[V_{k t}^{+*} V_{k t^{\prime}}^{-}-V_{k t}^{-*} V_{k t^{\prime}}^{+}\right] \\
& \left.+\boldsymbol{e}_{z}\left[V_{k t}^{+*} V_{k t^{\prime}}^{+}-V_{k t}^{-*} V_{k t^{\prime}}^{-}\right]\right\}
\end{aligned}
$$

(d) Pseudovector spin-kinetic density

$$
\begin{aligned}
\boldsymbol{T}_{m}(\boldsymbol{r}) & =\left[\left(\nabla \cdot \nabla^{\prime}\right) \boldsymbol{s}_{m}\left(\boldsymbol{r}, \boldsymbol{r}^{\prime}\right)\right]_{r=\boldsymbol{r}^{\prime}} \\
& =\sum_{t t^{\prime}} \boldsymbol{T}^{t t^{\prime}}(r z) \tau_{t^{\prime} t}^{m},
\end{aligned}
$$

where

$$
\begin{aligned}
\boldsymbol{T}^{t t^{\prime}}(r z)= & \sum_{k}\left\{\boldsymbol { e } _ { r } \left(\left[\partial_{r} V_{k t}^{+*}\right]\left[\partial_{r} V_{k t^{\prime}}^{-}\right]+\frac{\Lambda^{+} \Lambda^{-}}{r^{2}} V_{k t}^{+*} V_{k t^{\prime}}^{-}\right.\right. \\
& +\left[\partial_{z} V_{k t}^{+*}\right]\left[\partial_{z} V_{k t^{\prime}}^{-}\right]+\left[\partial_{r} V_{k t}^{-*}\right]\left[\partial_{r} V_{k t^{\prime}}^{+}\right] \\
& \left.+\frac{\Lambda^{-} \Lambda^{+}}{r^{2}} V_{k t}^{-*} V_{k t^{\prime}}^{+}+\left[\partial_{z} V_{k t}^{-*}\right]\left[\partial_{z} V_{k t^{\prime}}^{+}\right]\right) \\
& +i \boldsymbol{e}_{\phi}\left(\left[\partial_{r} V_{k t}^{+*}\right]\left[\partial_{r} V_{k t^{\prime}}^{-}\right]+\frac{\Lambda^{-} \Lambda^{+}}{r^{2}} V_{k t}^{+*} V_{k t^{\prime}}^{-}\right. \\
& +\left[\partial_{z} V_{k t}^{+*}\right]\left[\partial_{z} V_{k t^{\prime}}^{-}\right]-\left[\partial_{r} V_{k t}^{-*}\right]\left[\partial_{r} V_{k t^{\prime}}^{+}\right] \\
& \left.-\frac{\Lambda^{-} \Lambda^{+}}{r^{2}} V_{k t}^{-*} V_{k t^{\prime}}^{+}-\left[\partial_{z} V_{k t}^{-*}\right]\left[\partial_{z} V_{k t^{\prime}}^{+}\right]\right) \\
& +\boldsymbol{e}_{z}\left(\left[\partial_{r} V_{k t}^{+*}\right]\left[\partial_{r} V_{k t^{\prime}}^{+}\right]+\frac{\Lambda^{-2}}{r^{2}} V_{k t}^{+*} V_{k t^{\prime}}^{+}\right. \\
& +\left[\partial_{z} V_{k t}^{+*}\right]\left[\partial_{z} V_{k t}^{+}\right]-\left[\partial_{r} V_{k t}^{-*}\right]\left[\partial_{r} V_{k t^{\prime}}^{-}\right] \\
& \left.\left.-\frac{\Lambda^{+2}}{r^{2}} V_{k t}^{-*} V_{k t^{\prime}}^{-}-\left[\partial_{z} V_{k t}^{-*}\right]\left[\partial_{z} V_{k t^{\prime}}^{-}\right]\right)\right\}
\end{aligned}
$$

(e) Vector current density

$$
\begin{aligned}
\boldsymbol{j}_{m}(\boldsymbol{r}) & =\frac{1}{2 i}\left[\left(\nabla-\nabla^{\prime}\right) \rho_{m}\left(\boldsymbol{r}, \boldsymbol{r}^{\prime}\right)\right]_{\boldsymbol{r}=\boldsymbol{r}^{\prime}} \\
& =\sum_{t t^{\prime}} \boldsymbol{j}^{t t^{\prime}}(r z) \tau_{t^{\prime} t}^{m},
\end{aligned}
$$

where

$$
\begin{aligned}
\boldsymbol{j}^{t t^{\prime}}(r z)= & \frac{1}{2 i} \sum_{k}\left\{\boldsymbol { e } _ { r } \left(\left[\partial_{r} V_{k t}^{+*}\right] V_{k t^{\prime}}^{+}+\left[\partial_{r} V_{k t}^{-*}\right] V_{k t^{\prime}}^{-}\right.\right. \\
& \left.-V_{k t}^{+*}\left[\partial_{r} V_{k t^{\prime}}^{+}\right]-V_{k t}^{-*}\left[\partial_{r} V_{k t^{\prime}}^{-}\right]\right) \\
& -2 i \boldsymbol{e}_{\phi}\left(\frac{\Lambda^{-}}{r} V_{k t}^{+*} V_{k t^{\prime}}^{+}+\frac{\Lambda^{+}}{r} V_{k t}^{-*} V_{k t^{\prime}}^{-}\right) \\
& +\boldsymbol{e}_{z}\left(\left[\partial_{z} V_{k t}^{+*}\right] V_{k t^{\prime}}^{+}+\left[\partial_{z} V_{k t}^{-*}\right] V_{k t^{\prime}}^{-}\right. \\
& \left.\left.-V_{k t}^{+*}\left[\partial_{z} V_{k t^{\prime}}^{+}\right]-V_{k t}^{-*}\left[\partial_{z} V_{k t^{\prime}}^{-}\right]\right)\right\} .
\end{aligned}
$$

(f) Tensor spin current density

$$
\mathrm{J}_{m}^{i j}=\left.\frac{1}{2 i}\left(\nabla_{i}-\nabla_{i}^{\prime}\right) s_{m}^{j}\left(\boldsymbol{r}, \boldsymbol{r}^{\prime}\right)\right|_{\boldsymbol{r}=\boldsymbol{r}^{\prime}}=\sum_{t t^{\prime}} \mathrm{J}_{i j}^{t t^{\prime}}(r z) \tau_{t^{\prime} t}^{m} .
$$

Explicit expressions of the components are

$$
\begin{aligned}
J_{r \phi}^{t t^{\prime}}(r z)= & \frac{1}{2} \sum_{k}\left\{\left[\partial_{r} V_{k t}^{+*}\right] V_{k t^{\prime}}^{-}-V_{k t}^{+*}\left[\partial_{r} V_{k t^{\prime}}^{-}\right]\right. \\
& \left.-\left[\partial_{r} V_{k t}^{-*}\right] V_{k t^{\prime}}^{+}+V_{k t}^{-*}\left[\partial_{r} V_{k t^{\prime}}^{+}\right]\right\}, \\
J_{r z}^{t t^{\prime}}(r z)= & \frac{1}{2 i} \sum_{k}\left\{\left[\partial_{r} V_{k t}^{+*}\right] V_{k t^{\prime}}^{+}-V_{k t}^{+*}\left[\partial_{r} V_{k t^{\prime}}^{+}\right]\right.
\end{aligned}
$$$$
\left.-\left[\partial_{r} V_{k t}^{-*}\right] V_{k t^{\prime}}^{-}+V_{k t}^{-*}\left[\partial_{r} V_{k t^{\prime}}^{-}\right]\right\},
$$

$$
J_{\phi z}^{t t^{\prime}}(r z)=-\sum_{k}\left\{\frac{\Lambda^{-}}{r} V_{k t}^{+*} V_{k t^{\prime}}^{+}-\frac{\Lambda^{+}}{r} V_{k t}^{-*} V_{k t^{\prime}}^{-}\right\},
$$

$$
J_{z \phi}^{t t^{\prime}}(r z)=\frac{1}{2} \sum_{k}\left\{\left[\partial_{z} V_{k t}^{+*}\right] V_{k t^{\prime}}^{-}-V_{k t}^{+*}\left[\partial_{z} V_{k t^{\prime}}^{-}\right]\right.
$$$$
\left.-\left[\partial_{z} V_{k t}^{-*}\right] V_{k t^{\prime}}^{+}+V_{k t}^{-*}\left[\partial_{z} V_{k t^{\prime}}^{+}\right]\right\},
$$

$$
\mathrm{J}_{z r}^{t t^{\prime}}(r z)=\frac{1}{2 i} \sum_{k}\left\{\left[\partial_{z} V_{k t}^{+*}\right] V_{k t^{\prime}}^{-}-V_{k t}^{+*}\left[\partial_{z} V_{k t^{\prime}}^{-}\right]\right.
$$$$
\left.+\left[\partial_{z} V_{k t}^{-*}\right] V_{k t^{\prime}}^{+}-V_{k t}^{-*}\left[\partial_{z} V_{k t^{\prime}}^{+}\right]\right\},
$$

$$
J_{\phi r}^{t t^{\prime}}(r z)=-\frac{1}{2} \sum_{k}\left\{\frac{\Lambda^{-}}{r} V_{k t}^{+*} V_{k t^{\prime}}^{-}+\frac{\Lambda^{-}}{r} V_{k t}^{-*} V_{k t^{\prime}}^{+}\right.
$$$$
\left.+\frac{\Lambda^{+}}{r} V_{k t}^{+*} V_{k t^{\prime}}^{-}+\frac{\Lambda^{+}}{r} V_{k t}^{-*} V_{k t^{\prime}}^{+}\right\},
$$

$$
J_{z z}^{t t^{\prime}}(r z)=\frac{1}{2 i} \sum_{k}\left\{\left[\partial_{z} V_{k t}^{+*}\right] V_{k t^{\prime}}^{+}-V_{k t}^{+*}\left[\partial_{z} V_{k t^{\prime}}^{+}\right]\right.
$$$$
\left.-\left[\partial_{z} V_{k t}^{-*}\right] V_{k t^{\prime}}^{-}+V_{k t}^{-*}\left[\partial_{z} V_{k t^{\prime}}^{-}\right]\right\},
$$

$\mathrm{J}_{\phi \phi}^{t t^{\prime}}(r z)=-\frac{1}{2 i} \sum_{k}\left\{\frac{\Lambda^{-}}{r} V_{k t}^{-*} V_{k t^{\prime}}^{+}+\frac{\Lambda^{+}}{r} V_{k t}^{-*} V_{k t^{\prime}}^{+}\right.$

$$
\begin{aligned}
& \left.-\frac{\Lambda^{+}}{r} V_{k t}^{+*} V_{k t^{\prime}}^{-}-\frac{\Lambda^{-}}{r} V_{k t}^{+*} V_{k t^{\prime}}^{-}\right\}, \\
\mathrm{J}_{r r}^{t t^{\prime}}(r z)= & \frac{1}{2 i} \sum_{k}\left\{\left[\partial_{r} V_{k t}^{+*}\right] V_{k t^{\prime}}^{-}-V_{k t}^{+*}\left[\partial_{r} V_{k t^{\prime}}^{-}\right]\right. \\
& \left.+\left[\partial_{r} V_{k t}^{-*}\right] V_{k t^{\prime}}^{+}-V_{k t}^{-*}\left[\partial_{r} V_{k t^{+}}^{+}\right]\right\} .
\end{aligned}
$$

The trace, antisymmetric, and symmetric parts of the tensor spin-current density are given by

$$
\begin{aligned}
J_{k}(\boldsymbol{r}) & =\sum_{a=x, y, z} \mathrm{~J}_{k a a}(\boldsymbol{r}), \\
\boldsymbol{J}_{k a}(\boldsymbol{r}) & =\sum_{b, c=x, y, z} \boldsymbol{\epsilon}_{a b c} \mathrm{~J}_{k b c}(\boldsymbol{r}), \\
\underline{J}_{k a b}(\boldsymbol{r}) & =\frac{1}{2} \mathrm{~J}_{k a b}(\boldsymbol{r})+\frac{1}{2} \mathrm{~J}_{k b a}(\boldsymbol{r})-\frac{1}{3} J_{k}(\boldsymbol{r}) \delta_{a b} .
\end{aligned}
$$




\section{Derivatives of the densities}

(a) Divergence of tensor spin current density

$$
\begin{aligned}
{\left[\nabla \cdot \boldsymbol{J}_{m}(\boldsymbol{r})\right]=} & \frac{1}{2 i} \sum_{k s s^{\prime} t t^{\prime}}\left\{\nabla V_{k}\left(\boldsymbol{r} s^{\prime} t^{\prime}\right) \cdot\left(\nabla \times \hat{\boldsymbol{\sigma}}_{s^{\prime} s}\right) V_{k}^{*}(\boldsymbol{r} s t)\right. \\
& \left.-\nabla V_{k}^{*}(\boldsymbol{r} s t) \cdot\left(\nabla \times \hat{\boldsymbol{\sigma}}_{s^{\prime} s}\right) V_{k}\left(\boldsymbol{r} s^{\prime} t^{\prime}\right)\right\} \tau_{t^{\prime} t}^{m} \\
= & \sum_{t t^{\prime}}\left(D J_{r}^{t t^{\prime}}(r z)+D J_{\phi}^{t t^{\prime}}(r z)+D J_{z}^{t t^{\prime}}(r z)\right) \tau_{t^{\prime} t}^{m},
\end{aligned}
$$

where

$$
\begin{aligned}
D J_{r}^{t t^{\prime}}(r z)= & \frac{1}{2} \sum_{k}\left\{-\frac{\Lambda^{-}}{r}\left[\partial_{r} V_{k t^{\prime}}^{+}\right] V_{k t}^{+*}-\frac{\Lambda^{-}}{r}\left[\partial_{r} V_{k t}^{+*}\right] V_{k t^{\prime}}^{+}\right. \\
& +\left[\partial_{r} V_{k t}^{+*}\right]\left[\partial_{z} V_{k t^{\prime}}^{-}\right]-\left[\partial_{r} V_{k t}^{-*}\right]\left[\partial_{z} V_{k t^{\prime}}^{+}\right] \\
& -\left[\partial_{r} V_{k t^{\prime}}^{-}\right]\left[\partial_{z} V_{k t}^{+*}\right]+\left[\partial_{r} V_{k t^{\prime}}^{+}\right]\left[\partial_{z} V_{k t}^{-*}\right] \\
& \left.+\frac{\Lambda^{+}}{r}\left[\partial_{r} V_{k t^{\prime}}^{-}\right] V_{k t}^{-*}+\frac{\Lambda^{+}}{r}\left[\partial_{r} V_{k t}^{-*}\right] V_{k t^{\prime}}^{-}\right\}
\end{aligned}
$$

$$
\begin{aligned}
D J_{\phi}^{t t^{\prime}}(r z)= & -\frac{1}{2} \sum_{k}\left\{\frac{\Lambda^{-}}{r} V_{k t^{\prime}}^{+}\left[\partial_{r} V_{k t}^{+*}\right]+\frac{\Lambda^{-}}{r} V_{k t}^{+*}\left[\partial_{r} V_{k t^{\prime}}^{+}\right]\right. \\
& -\frac{\Lambda^{-}}{r} V_{k t^{\prime}}^{+}\left[\partial_{z} V_{k t}^{-*}\right]-\frac{\Lambda^{+}}{r} V_{k t}^{-*}\left[\partial_{z} V_{k t^{\prime}}^{+}\right] \\
& -\frac{\Lambda^{+}}{r} V_{k t^{\prime}}^{-}\left[\partial_{z} V_{k t}^{+*}\right]-\frac{\Lambda^{-}}{r} V_{k t}^{+*}\left[\partial_{z} V_{k t^{\prime}}^{-}\right] \\
& \left.-\frac{\Lambda^{+}}{r} V_{k t^{\prime}}^{-}\left[\partial_{r} V_{k t}^{-*}\right]-\frac{\Lambda^{+}}{r} V_{k t}^{-*}\left[\partial_{r} V_{k t^{\prime}}^{-}\right]\right\}
\end{aligned}
$$

$$
\begin{aligned}
D J_{z}^{t t^{\prime}}(r z)= & -\frac{1}{2} \sum_{k}\left\{\left[\partial_{z} V_{k t^{\prime}}^{+}\right]\left[\partial_{r} V_{k t}^{-*}\right]-\frac{\Lambda^{+}}{r}\left[\partial_{z} V_{k t^{\prime}}^{+}\right] V_{k t}^{-*}\right. \\
& -\left[\partial_{z} V_{k t}^{-*}\right]\left[\partial_{r} V_{k t^{\prime}}^{+}\right]-\frac{\Lambda^{-}}{r}\left[\partial_{z} V_{k t}^{-*}\right] V_{k t^{\prime}}^{+} \\
& -\left[\partial_{z} V_{k t^{\prime}}^{-}\right]\left[\partial_{r} V_{k t}^{+*}\right]-\frac{\Lambda^{-}}{r}\left[\partial_{z} V_{k t^{-}}^{-}\right] V_{k t}^{+*} \\
& \left.+\left[\partial_{z} V_{k t}^{+*}\right]\left[\partial_{r} V_{k t^{\prime}}^{-}\right]-\frac{\Lambda^{+}}{r}\left[\partial_{z} V_{k t}^{+*}\right] V_{k t^{\prime}}^{-}\right\} .
\end{aligned}
$$

Isospin components are given by

$$
\begin{aligned}
& \nabla \cdot \boldsymbol{J}_{0}(\boldsymbol{r})=\sum_{i=(r, \phi, z)}\left(D J_{i}^{n n}(r z)+D J_{i}^{p p}(r z)\right), \\
& \nabla \cdot \boldsymbol{J}_{1}(\boldsymbol{r})=\sum_{i=(r, \phi, z)}\left(D J_{i}^{n p}(r z)+D J_{i}^{p n}(r z)\right), \\
& \nabla \cdot \boldsymbol{J}_{2}(\boldsymbol{r})=i \sum_{i=(r, \phi, z)}\left(D J_{i}^{n p}(r z)-D J_{i}^{p n}(r z)\right),
\end{aligned}
$$

$$
\nabla \cdot \boldsymbol{J}_{3}(\boldsymbol{r})=\sum_{i=(r, \phi, z)}\left(D J_{i}^{n n}(r z)-D J_{i}^{p p}(r z)\right) .
$$

(b) Curl of current density

$$
\nabla \times \boldsymbol{j}_{m}(\boldsymbol{r})=\sum_{t t^{\prime}}(\nabla \times \boldsymbol{j})^{t t^{\prime}}(r z) \tau_{t^{\prime} t}^{m}
$$

where

$$
\begin{aligned}
(\nabla \times & \boldsymbol{j})^{t t^{\prime}}(r z) \\
= & \sum_{k}\left\{\boldsymbol { e } _ { r } \left(\frac{\Lambda^{-}}{r} V_{k t}^{+*}\left[\partial_{z} V_{k t^{+}}^{+}\right]+\frac{\Lambda^{+}}{r} V_{k t}^{-*}\left[\partial_{z} V_{k t^{-}}^{-}\right]\right.\right. \\
& \left.+\frac{\Lambda^{-}}{r}\left[\partial_{z} V_{k t}^{+*}\right] V_{k t^{\prime}}^{+}+\frac{\Lambda^{+}}{r}\left[\partial_{z} V_{k t}^{-*}\right] V_{k t^{\prime}}^{-}\right) \\
& +i \boldsymbol{e}_{\phi}\left(\left[\partial_{z} V_{k t}^{+*}\right]\left[\partial_{r} V_{k t^{\prime}}^{+}\right]+\left[\partial_{z} V_{k t}^{-*}\right]\left[\partial_{r} V_{k t^{\prime}}^{-}\right]\right. \\
& \left.-\left[\partial_{r} V_{k t}^{+*}\right]\left[\partial_{z} V_{k t^{\prime}}^{+}\right]-\left[\partial_{r} V_{k t}^{-*}\right]\left[\partial_{z} V_{k t^{\prime}}^{-}\right]\right) \\
& -\boldsymbol{e}_{z}\left(\frac{\Lambda^{-}}{r}\left[\partial_{r} V_{k t}^{+*}\right] V_{k t^{\prime}}^{+}+\frac{\Lambda^{+}}{r}\left[\partial_{r} V_{k t}^{-*}\right] V_{k t^{\prime}}^{-}\right. \\
& \left.\left.+\frac{\Lambda^{-}}{r} V_{k t}^{+*}\left[\partial_{r} V_{k t^{\prime}}^{+}\right]+\frac{\Lambda^{+}}{r} V_{k t}^{-*}\left[\partial_{r} V_{k t^{\prime}}^{-}\right]\right)\right\} .
\end{aligned}
$$

(c) Curl of spin density

$$
\nabla \times \boldsymbol{s}_{m}(\boldsymbol{r})=\sum_{t t^{\prime}}(\nabla \times s)^{t t^{\prime}}(r z) \tau_{t^{\prime} t}^{m}
$$

where

$$
\begin{aligned}
(\nabla \times s)^{t t^{\prime}}(r z)= & i \sum_{k}\left\{\boldsymbol { e } _ { r } \left(\left[\partial_{z} V_{k t}^{-*}\right] V_{k t^{\prime}}^{+}+V_{k t}^{-*}\left[\partial_{z} V_{k t^{\prime}}^{+}\right]\right.\right. \\
& \left.-\left[\partial_{z} V_{k t}^{+*}\right] V_{k t^{\prime}}^{-}-V_{k t}^{+*}\left[\partial_{z} V_{k t^{\prime}}^{-}\right]\right) \\
& -i \boldsymbol{e}_{\phi}\left(\left[\partial_{z} V_{k t}^{-*}\right] V_{k t^{\prime}}^{+}+V_{k t}^{-*}\left[\partial_{z} V_{k t^{\prime}}^{+}\right]\right. \\
& +\left[\partial_{z} V_{k t}^{+*}\right] V_{k t^{\prime}}^{-}+V_{k t}^{+*}\left[\partial_{z} V_{k t^{\prime}}^{-}\right] \\
& -\left[\partial_{r} V_{k t}^{+*}\right] V_{k t^{\prime}}^{+}-V_{k t}^{+*}\left[\partial_{r} V_{k t^{\prime}}^{+}\right] \\
& \left.+\left[\partial_{r} V_{k t}^{-*}\right] V_{k t^{\prime}}^{-}+V_{k t}^{-*}\left[\partial_{r} V_{k t^{\prime}}^{-}\right]\right) \\
& +\boldsymbol{e}_{z}\left(\left[\partial_{r} V_{k t}^{+*}\right] V_{k t^{\prime}}^{-}+V_{k t}^{+*}\left[\partial_{r} V_{k t^{\prime}}^{-}\right]\right. \\
& \left.\left.-\left[\partial_{r} V_{k t}^{-*}\right] V_{k t^{\prime}}^{+}-V_{k t}^{-*}\left[\partial_{r} V_{k t^{\prime}}^{+}\right]\right)\right\} .
\end{aligned}
$$

(d) Laplacian of $\rho$

$$
\nabla^{2} \rho_{m}(\boldsymbol{r})=\sum_{t t^{\prime}}\left(2 \tau^{t t^{\prime}}(r z)+L^{t t^{\prime}}(r z)\right) \tau_{t^{\prime} t}^{m}
$$

where

$$
\begin{aligned}
L^{t t^{\prime}}(r z)= & \sum_{k}\left\{\bar{V}_{k t}^{+*}\left[\nabla^{2} \bar{V}_{k t^{\prime}}^{+}\right]+\bar{V}_{k t}^{-*}\left[\nabla^{2} \bar{V}_{k t^{\prime}}^{-}\right]\right. \\
& \left.+\bar{V}_{k t^{\prime}}^{+}\left[\nabla^{2} \bar{V}_{k t}^{+*}\right]+\bar{V}_{k t^{\prime}}^{-}\left[\nabla^{2} \bar{V}_{k t}^{-*}\right]\right\}
\end{aligned}
$$




$$
\begin{aligned}
& \bar{V}_{k t}^{+}=V_{k t}^{+} e^{i \Lambda^{-} \phi}, \\
& \bar{V}_{k t}^{-}=V_{k t}^{-} e^{i \Lambda^{+} \phi} .
\end{aligned}
$$

(e) Laplacian of $\boldsymbol{s}$

$$
\begin{aligned}
\nabla^{2} \boldsymbol{s}_{m}(\boldsymbol{r})= & \sum_{t t^{\prime}}\left(2 \boldsymbol{T}^{t t^{\prime}}(r z)+\boldsymbol{S}^{t t^{\prime}}(r z)\right. \\
& \left.-\frac{\boldsymbol{e}_{r}}{r^{2}} s_{r}^{t t^{\prime}}(r z)-\frac{\boldsymbol{e}_{\phi}}{r^{2}} s_{\phi}^{t t^{\prime}}(r z)\right) \tau_{t^{\prime} t}^{m},
\end{aligned}
$$

where

$$
\begin{aligned}
\boldsymbol{S}^{t t^{\prime}}(r z)= & \sum_{k}\left\{\boldsymbol { e } _ { r } \left(\left[\nabla^{2} \bar{V}_{k t}^{-*}\right] \bar{V}_{k t^{\prime}}^{+}+\bar{V}_{k t}^{-*}\left[\nabla^{2} \bar{V}_{k t^{\prime}}^{+}\right]\right.\right. \\
& \left.+\left[\nabla^{2} \bar{V}_{k t}^{+*}\right] \bar{V}_{k t^{\prime}}^{-}+\bar{V}_{k t}^{+*}\left[\nabla^{2} \bar{V}_{k t^{\prime}}^{-}\right]\right) \\
& +i \boldsymbol{e}_{\phi}\left(-\left[\nabla^{2} \bar{V}_{k t}^{-*}\right] \bar{V}_{k t^{\prime}}^{+}-\bar{V}_{k t}^{-*}\left[\nabla^{2} \bar{V}_{k t^{\prime}}^{+}\right]\right. \\
& \left.+\left[\nabla^{2} \bar{V}_{k t}^{+*}\right] \bar{V}_{k t^{\prime}}^{-}+\bar{V}_{k t}^{+*}\left[\nabla^{2} \bar{V}_{k t^{\prime}}^{-}\right]\right) \\
& +\boldsymbol{e}_{z}\left(\left[\nabla^{2} \bar{V}_{k t}^{+*}\right] \bar{V}_{k t^{\prime}}^{+}+\bar{V}_{k t}^{+*}\left[\nabla^{2} \bar{V}_{k t^{\prime}}^{+}\right]\right. \\
& \left.\left.-\left[\nabla^{2} \bar{V}_{k t}^{-*}\right] \bar{V}_{k t^{\prime}}^{-}-\bar{V}_{k t}^{-*}\left[\nabla^{2} \bar{V}_{k t^{\prime}}^{-}\right]\right)\right\} .
\end{aligned}
$$

[1] M. Stoitsov, M. Kortelainen, S. K. Bogner, T. Duguet, R. J. Furnstahl, B. Gebremariam, and N. Schunck, Phys. Rev. C 82, 054307 (2010).

[2] J. Dobaczewski, K. Bennaceur, and F. Raimondi, J. Phys. G 39, 125103 (2012).

[3] S. Bogner et al., Comput. Phys. Commun. 184, 2235 (2013).

[4] M. Kortelainen, J. McDonnell, W. Nazarewicz, E. Olsen, P. G. Reinhard, J. Sarich, N. Schunck, S. M. Wild, D. Davesne, J. Erler, and A. Pastore, arXiv:1312.1746.

[5] W. Satuła, R. A. Wyss, and M. Rafalski, Phys. Rev. C 74, 011301(R) (2006).

[6] A. Afanasjev, in Fifty Years of Nuclear BCS, edited by R. A. Broglia and V. Zelevinsky (World Scientific, Singapore, 2013), p. 138.

[7] N. Zeldes, in Handbook of Nuclear Properties, edited by D. Poenaru and W. Greiner (Clarendon, Oxford, 1996), p. 13.

[8] W. Satuła, D. J. Dean, J. Gary, S. Mizutori, and W. Nazarewicz, Phys. Lett. B 407, 103 (1997).

[9] A. L. Goodman, in Advances in Nuclear Physics, Vol. 11, edited by J. V. Negele and E. Vogt (Plenum Press, New York, 1979), p. 263.

[10] J. Terasaki, R. Wyss, and P.-H. Heenen, Phys. Lett. B 437, 1 (1998).

[11] S. G. Frauendorf and J. A. Sheikh, Nucl. Phys. A 645, 509 (1999).

[12] J. A. Sheikh and R. Wyss, Phys. Rev. C 62, 051302(R) (2000).

[13] W. Satuła and R. A. Wyss, Acta Phys. Pol. B 32, 2441 (2001).

[14] A. Gezerlis, G. F. Bertsch, and Y. L. Luo, Phys. Rev. Lett. 106, 252502 (2011).

[15] S. G. Rohoziński, J. Dobaczewski, and W. Nazarewicz, Phys. Rev. C 81, 014313 (2010).

[16] L. M. Robledo and G. F. Bertsch, Phys. Rev. C 84, 014312 (2011).

[17] E. Perlińska, S. G. Rohoziński, J. Dobaczewski, and W. Nazarewicz, Phys. Rev. C 69, 014316 (2004).

[18] M. V. Stoitsov, J. Dobaczewski, W. Nazarewicz, S. Pittel, and D. J. Dean, Phys. Rev. C 68, 054312 (2003).

[19] J. Erler, N. Birge, M. Kortelainen, W. Nazarewicz, E. Olsen, A. M. Perhac, and M. Stoitsov, Nature (London) 486, 509 (2012).

[20] M. Kortelainen, J. Erler, W. Nazarewicz, N. Birge, Y. Gao, and E. Olsen, Phys. Rev. C 88, 031305(R) (2013).

[21] E. Olsen, M. Pfützner, N. Birge, M. Brown, W. Nazarewicz, and A. Perhac, Phys. Rev. Lett. 110, 222501 (2013); 111, 139903(E) (2013).
[22] M. V. Stoitsov, J. Dobaczewski, W. Nazarewicz, and P. Ring, Comput. Phys. Commun. 167, 43 (2005).

[23] M. V. Stoitsov, N. Schunck, M. Kortelainen, N. Michel, H. Nam, E. Olsen, J. Sarich, and S. Wild, Comput. Phys. Commun. 184, 1592 (2013).

[24] M. Kortelainen, T. Lesinski, J. Moré, W. Nazarewicz, J. Sarich, N. Schunck, M. V. Stoitsov, and S. Wild, Phys. Rev. C 82, 024313 (2010); M. Kortelainen, J. McDonnell, W. Nazarewicz, P.-G. Reinhard, J. Sarich, N. Schunck, M. V. Stoitsov, and S. M. Wild, ibid. 85, 024304 (2012).

[25] K. Sato, J. Dobaczewski, T. Nakatsukasa, and W. Satuła, Phys. Rev. C 88, 061301(R) (2013).

[26] N. Schunck, J. Dobaczewski, J. McDonnell, W. Satuła, J. A. Sheikh, A. Staszczak, M. Stoitsov, and P. Toivanen, Comput. Phys. Commun. 183, 166 (2012). N. Schunck et al. (unpublished).

[27] P. Ring and P. Schuck, The Nuclear Many-Body Problem (Springer-Verlag, Berlin, 1980).

[28] J. Dobaczewski, W. Nazarewicz, T. R. Werner, J. F. Berger, C. R. Chinn, and J. Dechargé, Phys. Rev. C 53, 2809 (1996).

[29] W. Satuła and R. Wyss, Phys. Rev. Lett. 86, 4488 (2001); 87, 052504 (2001).

[30] J. Bartel, P. Quentin, M. Brack, C. Guet, and H.-B. Håkansson, Nucl. Phys. A 386, 79 (1982).

[31] C. A. Engelbrecht and R. H. Lemmer, Phys. Rev. Lett. 24, 607 (1970).

[32] N. Auerbach, Phys. Rep. 98, 273 (1983).

[33] W. Satuła, J. Dobaczewski, W. Nazarewicz, and M. Rafalski, Phys. Rev. Lett. 103, 012502 (2009).

[34] B. G. Carlsson, J. Dobaczewski, and M. Kortelainen, Phys. Rev. C 78, 044326 (2008).

[35] W. Satuła, J. Dobaczewski, W. Nazarewicz, and M. Rafalski, Phys. Rev. C 81, 054310 (2010).

[36] S. Shlomo, Rep. Prog. Phys. 41, 957 (1978).

[37] W. Nazarewicz, J. Dobaczewski, T. R. Werner, J. A. Maruhn, P.-G. Reinhard, K. Rutz, C. R. Chinn, A. S. Umar, and M. R. Strayer, Phys. Rev. C 53, 740 (1996).

[38] A. T. Kruppa and W. Nazarewicz, Phys. Rev. C 69, 054311 (2004).

[39] K. Bennaceur and J. Dobaczewski, Comput. Phys. Commun. 168, 96 (2005).

[40] T. Vertse, A. T. Kruppa, and W. Nazarewicz, Phys. Rev. C 61, 064317 (2000). 